\title{
Effects of Controlled Burning of Chaparral on Streamflow and Sediment Characteristics, East Fork Sycamore Creek, Central Arizona By STANLEY BALDYS III and H.W. HJALMARSON
}

U.S. GEOLOGICAL SURVEY

Water-Resources Investigations Report 93-4102

Prepared in cooperation with the SALT RIVER PROJECT and the U.S. FOREST SERVICE

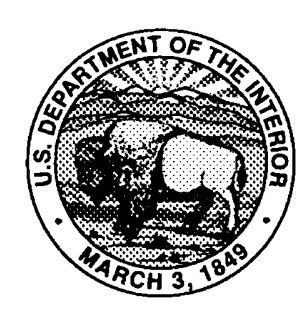




\section{U.S. DEPARTMENT OF THE INTERIOR BRUCE BABBITT, Secretary}

U.S. GEOLOGICAL SURVEY

Robert M. Hirsch, Acting Director

For additional information write to:

District Chief

U.S. Geological Survey Water Resources Division 375 South Euclid Avenue Tucson, AZ 85719-6644
Copies of this report can be purchased from:

U.S. Geological Survey

Earth Science Information Center Open-File Reports Section Box 25286, MS 517 Denver Federal Center Denver, CO 80225 


\section{CONTENTS}

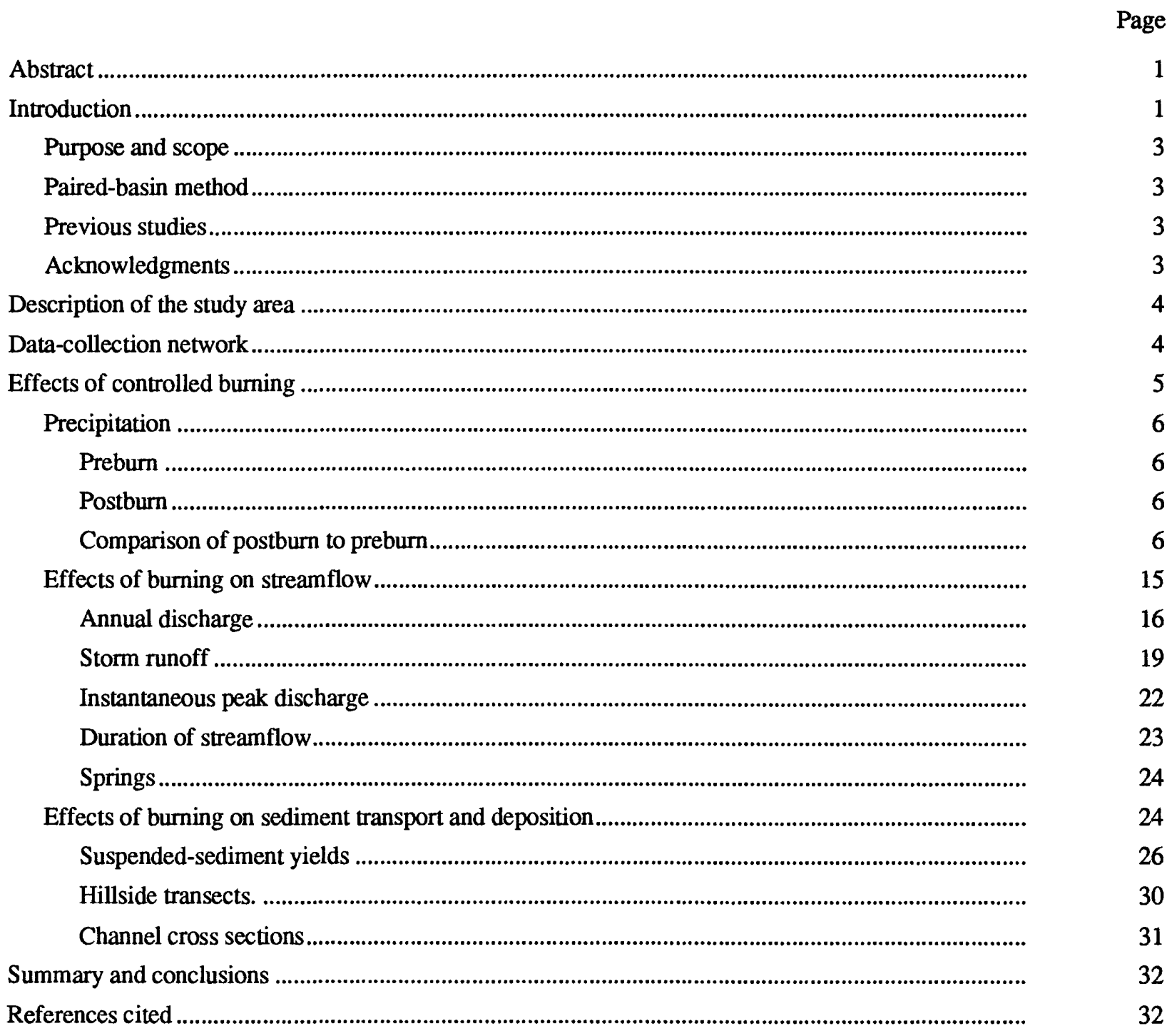

\section{ILLUSTRATIONS}

Plate 1. Map showing data-collection network and area burned by fire.

\section{FIGURES}

1. Map showing location of study area of this report and selected chaparral studies in Arizona. 


\section{FIGURES}

2-8. Graphs showing:

2. Annual precipitation for water years $1966-73$ and estimated annual precipitation for water years 1983-86, East Fork and West Fork Sycamore Creek drainage basins....

3. Monthly precipitation as a percentage of average annual precipitation, East Fork and West Fork Sycamore Creek drainage basins, water years 1966-73

4. Annual discharge in East Fork Sycamore Creek drainage basin and annual discharge in West Fork Sycamore Creek drainage basin, water years 1966-73 and 1983-86

5. Postburn storm runoff in East Fork and West Fork Sycamore Creek drainage basins, water years 1983-86

6. Flow-duration curves, East Fork and West Fork Sycamore Creek, water years 1966-73 and 1983-84.

7. Streamflow and suspended-sediment concentrations, East Fork and West Fork Sycamore Creek, February 8, 1983

8. Mean suspended-sediment yield in the Three Bar experimental watershed before and after controlled burning in 1959 and mean suspended-sediment yield on East Fork Sycamore Creek after wildfire in 1981

\section{TABLES}

1. Estimated daily precipitation, East Fork and West Fork Sycamore Creek drainage basins, water years 1983-86

2. Measured and computed annual discharge, East Fork and West Fork Sycamore Creek drainage basins, water years 1983-86.

3. Measured and computed streamflow for selected postburn periods of storm runoff, precipitation totals, and statistical-significance levels, East Fork and West Fork Sycamore Creek drainage basins, October 1982 through May 1986

4. Selected peak discharges and peak yields at the streamflow-gaging stations, East Fork and West Fork Sycamore Creek drainage basins, water years 1965-86

5. Range in measured discharge at Thicket and Oneda Mine Springs and Horse Camp Seep.

6. Annual suspended-sediment loads and yields, East Fork and West Fork Sycamore Creek drainage basins, water years 1983-86

7. Aggradation and degradation of colluvium, East Fork and West Fork Sycamore Creek drainage basins, water years 1983-85

8. Changes in cross-sectional area at channel-change monitoring sites, East Fork and

West Fork Sycamore Creek drainage basins and adjacent areas for indicated water years 
CONVERSION FACTORS AND VERTICAL DATUM

$\begin{array}{lcl}\text { Multiply } & \text { By } & \text { To obtain } \\ \text { inch (in.) } & 25.4 & \text { millimeter } \\ \text { foot (ft) } & 0.3048 & \text { meter } \\ \text { square foot }\left(\mathrm{ft}^{2}\right) & 0.0929 & \text { square meter } \\ \text { mile (mi) } & 1.609 & \text { kilometer } \\ \text { acre } & 0.4047 & \text { hectare } \\ \text { acre-foot (acre- } \mathrm{ft} \text { ) } & 0.001233 & \text { cubic hectometer } \\ \text { cubic foot per second }\left(\mathrm{ft}^{3} / \mathrm{s}\right) & 0.02832 & \text { cubic meter per second } \\ \text { cubic foot per acre }\left(\mathrm{ft}^{3} / \mathrm{acre}\right) & 0.06997 & \text { cubic meter per hectare } \\ \text { pound per cubic foot }\left(\mathrm{lb} / \mathrm{ft}^{3}\right) & 0.01284 & \text { kilogram per cubic meter } \\ \text { ton, short } & 907.2 & \text { kilogram } \\ \text { ton per square mile } & 350.4 & \text { kilogram per square } \\ \quad \text { ton } / \mathrm{mi}^{2} \text { ) } & & \text { kilometer }\end{array}$

Air temperatures are given in degrees Celsius $\left({ }^{\circ} \mathrm{C}\right)$, which can be converted to degrees Fahrenheit $\left({ }^{\circ} \mathrm{F}\right)$ by the following equation:

$$
{ }^{\circ} \mathrm{F}=1.8 \times^{\circ} \mathrm{C}+32
$$

Chemical concentration and water temperature are given only in metric units. Chemical concentration in water is given in milligrams per liter $(\mathrm{mg} / \mathrm{L})$ or micrograms per liter $(\mu \mathrm{g} / \mathrm{L})$. Milligrams per liter is a unit expressing the solute per unit volume (liter) of water. One thousand micrograms per liter is equivalent to 1 milligram per liter. For concentrations less than 7,000 milligrams per liter, the numerical value is about the same as for concentrations in parts per million. Chemical concentration in material from core samples is given in grams per kilogram ( $\mathrm{g} / \mathrm{kg}$ ) or micrograms per gram $(\mu \mathrm{g} / \mathrm{g})$. Micrograms per gram is equivalent to parts per million. Chemical concentration in material from core samples is given in grams per kilogram $(\mathrm{g} / \mathrm{kg})$ or micrograms per gram $(\mu \mathrm{g} / \mathrm{g})$. Micrograms per gram is equivalent to parts per million.

Sea level: In this report "sea level" refers to the National Geodetic Vertical Datum of 1929-a geodetic datum derived from a general adjustment of the first-order level nets of the United States and Canada, formerly called Sea Level Datum of 1929. 


\title{
Effects of Controlled Burning of Chaparral on Streamflow and Sediment Characteristics, East Fork Sycamore Creek, Central Arizona
}

\author{
By Stanley Baldys III and H.W. Hjalmarson
}

\begin{abstract}
A paired-basin method for determining changes in streamflow and suspended-sediment yield showed that the burning of chaparral vegetation in 45 percent of the East Fork Sycamore Creek basin in central Arizona increased the average annual streamflow by 14 percent for 2 years. The increase of streamflow for the 4.52-square-mile basin was greater for small storms than for large storms, and no change in flood-peak discharge was detected. A visual examination of preburn- and postburn-duration curves of daily discharge for the two basins showed an increase of flow over most of the range of streamflow. The flow of the East Fork basin changed from ephemeral to perennial during the second and third years following the burn. The study started 11 months following the controlled burn. Streamflow quantities appeared to return to preburn levels 3 years after the burn.
\end{abstract}

Changes in soil erosion as a result of the burn along hillside transects were not detected. Changes in channel aggradation and (or) degradation at cross sections along the main channels of both basins also were not detected. Because analysis of covariance of suspended-sediment yield of the paired basins indicates that the increase in suspended-sediment yield is related to the increase in streamflow and not to an increase in sediment concentration, the suspended-sediment yield was considered to return to preburn levels 3 years after the burning of the chaparral vegetation.

\section{INTRODUCTION}

Water consumption in central Arizona by public, agricultural, and industrial users has exceeded the natural quantities of water available from ground-water and surface-water sources (Thomsen and Baldys, 1985). Even with importation of water from the Colorado River by the Central Arizona Project, there is a water-supply deficit and water stored in aquifers is being used to meet present needs. One potential method to augment water supplies is to increase water yields from drainage basins by reducing transpiration through controlled burning of vegetation.

The Salt River and Verde River drainage basins, which have extensive areas of native chaparral, are the main sources of surface water for central Arizona. Studies by the U.S. Forest Service
(Pase and Ingebo, 1965) indicate that the conversion of chaparral to grasses or similar types of vegetation could substantially increase the quantity of water yield by reducing evapotranspiration. Studies also show that the sediment yield of basins with recently burned vegetation is large. The U.S. Geological Survey, in cooperation with the Salt River Project and the U.S. Forest Service, began a study of the effects of controlled burning of chaparral vegetation on streamflow and sediment yields. The study is on a tributary to the Verde River in central Arizona in a region where similar studies (shown as open areas on fig. 1) have been made.

Data collection for the study started in October 1965 when precipitation, streamflow, and sediment data were collected on two adjacent basins. The basins have similar physiography, topography, and 


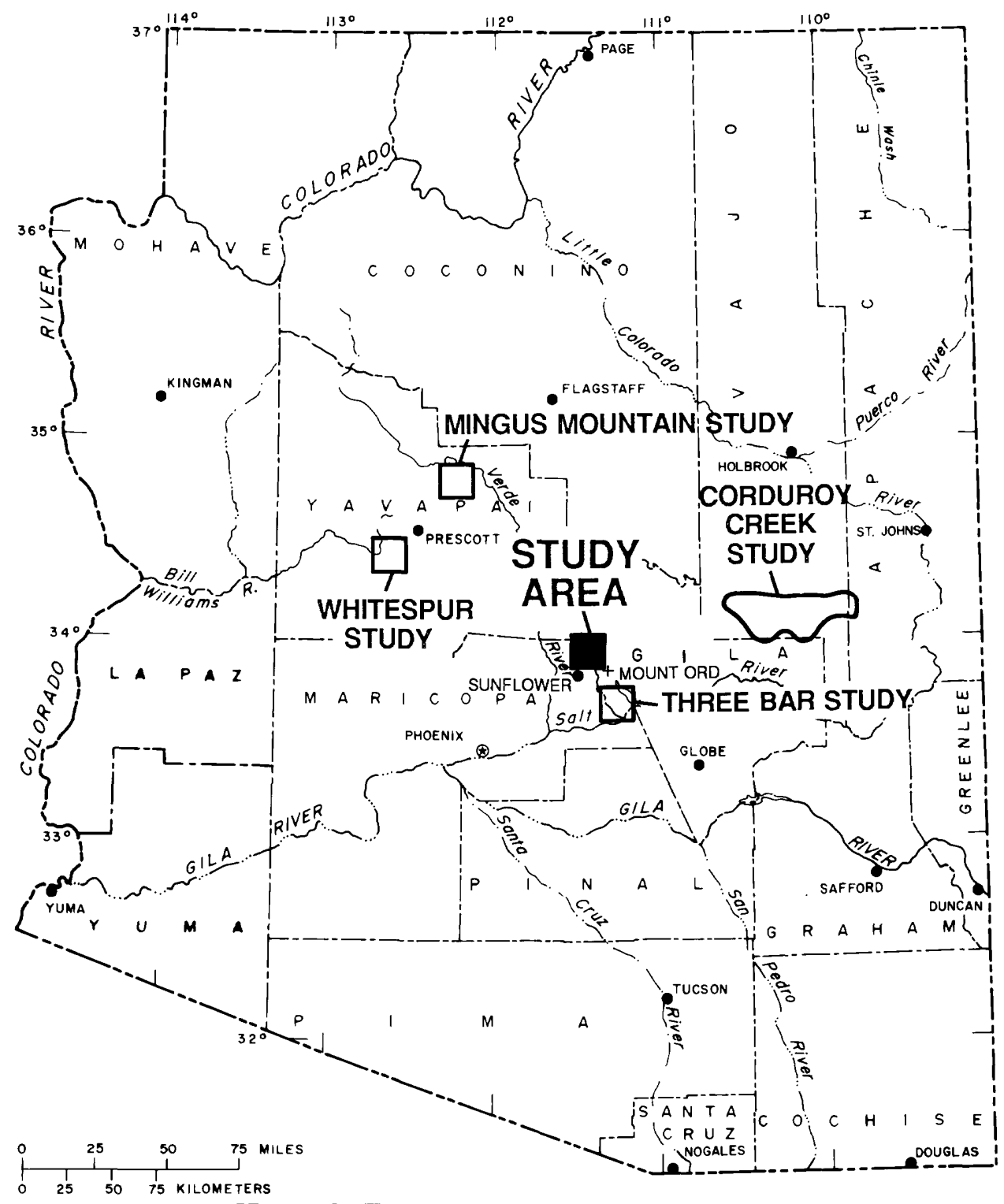

Flgure 1. Location of study area of this report and selected chaparral studies in Arizona.

vegetation, and the drainage areas are each about $4.6 \mathrm{mi}^{2}$. The dense chaparral vegetation of the East Fork Sycamore Creek basin was to be burned, and the vegetation of the West Fork Sycamore Creek basin was to remain undisturbed. After 8 years of data collection, the pair of basins was considered to be sufficiently calibrated to enable detection of changes in water and sediment yield. The vegetation could not be burned at that time, however, and the study was discontinued in
September 1973 (Hjalmarson, 1975). On October 31,1981 , about 45 percent of the vegetation of the East Fork basin was burned by the U.S. Forest Service. About 11 months after the controlled burn, the data collection was reactivated on October 1 , 1982. Data for the postburn conditions were collected through May 31, 1986, when the study was discontinued because the runoff and sediment yield appeared to have returned to preburn levels. 


\section{Purpose and Scope}

This report describes and analyzes the hydrologic data collected during the postburn period and analyzes the effect of the burned chaparral vegetation on the water and sediment yield of the East Fork basin. Two questions are addressed in the analysis: (1) Did changes take place in runoff and sediment yield, and if so, can the changes be attributed to the burning of the vegetation? (2) If a change in runoff and sediment yield did occur, how much was the change? At the core of the analysis is the comparison of preburn and postburn runoff and the sediment-yield relations using statistical methods. Analysis was made of the annual runoff, storm runoff, and flood-peak discharge of the two basins and spring flow in the basins. Annual sediment yield of the basins, aggradation and (or) degradation of colluvium in the basins, and changes in channel cross-section area in and downstream from the basins also were analyzed.

\section{Paired-Basin Method}

A paired-basin method was used for determining changes in runoff and suspended-sediment yield resulting from the burning of vegetation. The method uses statistical techniques to compare runoff and sediment yield of a control basin and an experimental basin, which normally receive similar amounts of precipitation. The West Fork drainage basin (control basin) and the East Fork drainage basin (experimental basin) share a common drainage boundary and have similar mean elevations and aspects. Precipitation amounts and intensities are similar for both basins for most storms (pl. 1, this report; Hjalmarson, 1975). The paired-basin method can produce reliable results if the physiography, topography, vegetation, geology, and soils of the basins are similar. Basins with similar hydrologic characteristics are more likely to produce similar rainfall-runoff relations; therefore, differences in streamflow and suspended-sediment yield following the controlled burn are the result of the burn and not dissimilar hydrologic characteristics.
An important feature of the paired-basin method is the rapid detection of hydrologic changes as a result of the burning and regrowth of the chaparral vegetation. Because the detection of changes in annual flows may take several years, streamflow data from storms were used as the test variable. This procedure shortened the time of data collection needed for reliable detection and allowed a more detailed examination of streamflow changes. A hydrograph-separation technique was used to determine independent runoff events.

\section{Previous Studies}

Several investigations have been made to determine changes in streamflow characteristics and sediment yield caused by various types of management practices of chaparral vegetation. In Arizona, studies have been done of the Three Bar watersheds (Pase and Ingebo, 1965), Mingus Mountain and Whitespar watersheds (Hibbert and others, 1975; Hibbert, 1981; Hibbert and Ingebo, 1971), and Corduroy Creek basin (Collings and Myrick, 1966; fig. 1, this report). One of several studies of chaparral-covered drainage basins in southern California was described by DeBano (1981).

The hydrologic characteristics of the Sycamore Creek drainage basin in central Arizona were described by Schumann (1967) and by Thomsen and Schumann (1968). The datacollection network, techniques used for the analysis, and data collected for the preburn period of this study were presented by Hjalmarson (1975).

\section{Acknowledgments}

The authors gratefully acknowledge the assistance of David Nelson and Richard C. Martin, Tonto National Forest, who surveyed the channel cross sections and hillside transects. Joseph B. Ryan, Jr., Rocky Mountain Forest and Range Experimental Station, analyzed suspendedsediment samples for the postburn period of the study. 


\section{DESCRIPTION OF THE STUDY AREA}

The drainage basins of West Fork Sycamore Creek above McFarland Canyon and East Fork Sycamore Creek near Sunflower are in Maricopa County about $50 \mathrm{mi}$ northeast of Phoenix (fig. 1). The drainage basins are similar and are on the southern slope of Mount Peeley between Mount Peeley and Mount Ord. The basins consist of rugged mountainous terrain with steep slopes. The median altitude is about $5,400 \mathrm{ft}$ above sea level for the West Fork drainage basin and 5,300 ft for the East Fork drainage basin.

The topographic, geologic, vegetative, and climatic characteristics of the two drainage basins are described in the report of the calibration or preburn period of the study (Hjalmarson, 1975, p. 6). Hjalmarson found that the topography, physiography, geology, soils, and vegetation of the two basins were similar and probably would not be factors to consider in the analysis of the effects of vegetation conversion on streamflow and sediment yields.

On the basis of more detailed topographic maps, the drainage areas of both basins were revised. The drainage area for the West Fork basin is $4.64 \mathrm{mi}^{2}$ (previously published as $4.58 \mathrm{mi}^{2}$ ), and the drainage area for the East Fork basin is $4.52 \mathrm{mi}^{2}$ (previously published as $4.49 \mathrm{mi}^{2}$ ).

During the preburn data-collection period, there was vehicular traffic associated with mining activity on roads in and crossing the main channels of both basins. During the postburn period, the mining activity in the area had ceased, and maintenance of access roads was limited.

The controlled burn by the U.S. Forest Service was confined to the upper two-thirds of the East Fork basin (pl. 1). A small area (3 percent) in the northeast corner of the West Fork basin also was inadvertently burned. The fire was started above the main channel of East Fork Sycamore Creek to avoid harming the large riparian vegetation on the banks. The fire crowned out and completely engulfed small ponderosa pine and juniper trees in most of the burn area. The smaller shrubs-manzanita, sugar sumac, and shrub oak-on the side slopes were completely consumed by the blaze.
The small area burned in the West Fork drainage basin was high in the basin upslope from any large stream channel (pl.1). Because the burned area was only 3 percent of the drainage area and near the drainage-basin divide, effects of the burn on streamflow and sediment yield at the gaging station were assumed to be insignificant and were not studied.

During October 1974, a 100-acre area (3.4 percent) of the West Fork drainage basin on the summit of Mount Peeley was burned by a wildfire named the Peeley Fire (David Nelson, hydrologist, U.S. Forest Service, Tonto National Forest, written commun., 1986). The Peeley Fire occurred early in the break between the two data-collection periods and should not affect the results and conclusions of the study. According to a fire atlas compiled by the Tonto National Forest (Richard C. Martin, hydrologist, U.S. Forest Service, Tonto National Forest, oral commun., 1987), there were no other recent large fires in the basins.

\section{DATA-COLLECTION NETWORK}

The data-collection network for the preburn period of the study consisted of 9 continuousrecording precipitation gages, 2 streamflow-gaging stations, 2 suspended-sediment sampling sites, 19 channel-change monitoring sites, 3 hillside transects for measuring colluvial aggradation and degradation, 2 spring-flow measuring sites, and 1 spring-flow gaging station (pl. 1). The network lies within the boundaries of the East Fork and West Fork drainage basins except for 15 channel-monitoring sites downstream from the basins.

The data-collection network was reinstrumented following the burn with two changes. The first change was to replace the nine precipitation gages with two gages-one in each drainage basin. For economic reasons, the number of gages could be reduced because statistical testing of precipitation data from the preburn period showed no significant difference in annual totals between the drainage basins (Hjalmarson, 1975, p. 24); therefore, precipitation amounts were not included as a variable in the regression equations. 
The second change was the replacement of the fixed-stage sediment samplers with stage-activated sediment samplers at the streamflow-gaging station capable of collecting discrete samples at predetermined sampling intervals. The suspended-sediment concentrations recorded during the preburn period varied widely for individual stages because the sampler could not collect discrete samples during rising or falling stream stages.

At the start of the postburn period, the two streamflow-gaging stations were instrumented with stage-activated pumping samplers capable of withdrawing discrete samples through an orifice at a fixed point during periods of runoff. The samples were referenced to cross-section samples manually collected using a depth-integrating sampler. A coefficient was determined and applied to suspended-sediment concentrations in the calculation of suspended-sediment yields.

Two types of automatic samplers were used during the postburn period. The first type was replaced in August 1983 by a sampler with a higher efficiency rating. This second type, which had a larger pump and a longer purge cycle before and after the sample was collected, allowed a more representative sample to be collected.

The two continuous streamflow-gaging stations used during the preburn period were reactivated. The structures that controlled the stage-discharge relations at the stations during the preburn period also were used during the postburn period.

Of the 19 original channel-change monitoring sites, 13 were retained during the postburn period. Sites 5 and 7 (pl. 1) were discontinued because of bulldozer activity in the area. Sites 1-3, below the confluence of East Fork and West Fork Sycamore Creek, were not surveyed during 1982-86 because of lack of changes in upstream sites. Site 13 was not resurveyed until October 1985 because of problems identifying its proper location.

The three hillside transects, which were used for measuring colluvial aggradation and degradation, were used during the postburn period without difficulty. Transect 1 is in the West Fork drainage basin, and transects 2 and 3 are in the East
Fork drainage basin (pl. 1). Each hillside transect is a 285-foot-long line with measuring points every $15 \mathrm{ft}$ where the amount of aggradation or degradation of the ground surface is determined. The determination is made by measuring the change in distance between the midpoint of a level laid on the tops of two steel rods and the ground surface perpendicular to the midpoint of the level at each measuring point. The hillside transects start near the top of a hill and proceed down the hill with no abrupt changes in slope.

Data collection at Horse Camp Seep (pl.1) was reduced from a continuous-record spring-flow gaging station to a measurement made once a year. Horse Camp Seep is upstream from the burn area, and flow from the spring should not have been affected by the burn. No change was made in data collection at Thicket Spring and Oneda Mine Spring.

\section{EFFECTS OF CONTROLLED BURNING}

Controlled burning of a drainage basin can severely affect streamflow characteristics and suspended-sediment yields. The burning of the vegetation and litter cover from a drainage basin removes the protective canopy that shields the soil from direct contact with precipitation and wind. The lack of the protective canopy can result in increased soil movement and runoff. Water that normally would have infiltrated into the soil and been used by the vegetation becomes runoff.

Streamflow and suspended-sediment yields can be increased by the formation of a hydrophobic soil layer produced by the burning of the vegetation and litter layer (DeBano, 1981, p. 5). Water repellency, produced by soil heating, is common on burned watersheds in southern California (DeBano, 1981 , p. 2). Fire heats organic particles in the soil to such an extent that they form a chemically bonded coat on the nearby mineral particles, which repels infiltration (DeBano, 1981, p. 5). The length of time that the water-repellent zone exists is not well known and is dependent on the physical and chemical properties of the hydrophobic soil layer, steepness of slope, intensity and amount of rainfall, 
conditions during the burn, and temperature of burn. Precipitation for each basin was examined first because differences in precipitation for the two basins can have an effect on the analysis of runoff and sediment yield.

\section{Precipitation}

Daily values of precipitation for each basin were computed for both the preburn and postburn periods. For the preburn period, the average daily precipitation for each basin was computed using the Theissen polygon method (Hjalmarson, 1975; Linsley and others, 1982). For the postburn period, the values were computed from continuous records of precipitation at the two gages near the center of the lower half of each basin (p1. 1). The daily values for the two gages were adjusted using a relation between altitude and precipitation defined during the preburn period by Hjalmarson (1975; table 1 , this report). The daily values computed using the two methods are assumed to be comparable and were summed to determine the annual precipitation for each basin (fig. 2).

\section{Preburn}

The West Fork basin received 3.6 percent more precipitation than the East Fork basin during the 8-year preburn period from 1965 to 1973 (Hjalmarson, 1975, p. 23). Computed precipitation for West Fork basin ranged from a low of $12.41 \mathrm{in}$. for water year 1972 to a high of 42.97 in. for water year 1973. The average annual precipitation was 26.1 in. for the preburn period. Computed annual precipitation for East Fork basin ranged from a low of $12.71 \mathrm{in}$. for water year 1972 to a high of 41.35 in. for water year 1973. The average annual precipitation was 25.2 in. for the 8 -year period (Hjalmarson, 1975).

\section{Postburn}

During the postburn period, the West Fork basin also received more precipitation than the East Fork basin (fig. 2). The average annual precipitation was 13.0 percent more for the West Fork basin than for the East Fork basin. Computed annual precipitation for the West Fork basin ranged from a low of $21.62 \mathrm{in}$. for 1984 to a high of $46.32 \mathrm{in}$. for
1983. The computed annual precipitation for the East Fork basin ranged from a low of 19.44 in. for 1984 to a high of $41.88 \mathrm{in}$. for 1983 . The average annual precipitation for the postburn period, 1983-85, was 34.8 in. for the West Fork basin and 30.8 in. for the East Fork basin. Although the computed average annual precipitation during preburn and postburn periods was more for the West Fork basin than for the East Fork basin, the difference was not statistically significant using a students' paired T-test (Inman and Conover, 1983, p. 246). In this study, therefore, annual precipitation was considered to be equal for the two basins.

Although precipitation data were not collected in the study area immediately after the fire of October 1981 through the rest of water year 1982 , data were available from the nearby climatic station at Sunflower, Arizona. The recorded amount of 19.5 in. was close to an 18-year average of 20.8 in. for that site (Sellers and Hill, 1974, p. 494).

The precipitation total of 17.04 in. recorded at the East Fork gage from October 1, 1985, through May 31, 1986, was projected to an annual total of 26.05 in. This estimate was made using the distribution of rainfall by month during the preburn period (fig. 3) and was just slightly greater than the preburn-period mean. The precipitation total of 19.80 in. recorded at the West Fork gage from October 1, 1985, through May 31, 1986, was projected to an annual total of $30.27 \mathrm{in}$. using the same procedure.

\section{Comparison of Postburn to Preburn}

The computed average annual precipitation for the postburn period is greater than that for the preburn period for both basins. The largest annual precipitation occurred in water year 1983 and was 46.32 and 41.88 in. for the West Fork and East Fork basins, respectively (fig. 2). These amounts are more than two standard deviations above the average annual precipitation for the preburn period. The large computed amount of annual precipitation for 1983, which was above the range of the annual precipitation for the preburn period, exemplifies the importance of selecting paired basins with similar physical characteristics as previously discussed. If the basins were dissimilar, the large amount of 
Table 1. Estimated daily precipitation, East Fork and West Fork Sycamore Creek drainage basins, water years 1983-86

[Dashes indicate no day]

09510099 East Fork Sycamore Creek near Sunflower

Rainfall accumulated, In Inches, water year October 1982 to September 1983

\begin{tabular}{|c|c|c|c|c|c|c|c|c|c|c|c|c|}
\hline Day & Oct. & Nov. & Dec. & Jan. & Feb. & Mar. & Apr. & May & June & July & Aug. & Sept. \\
\hline 1 & 0.00 & 0.00 & 0.00 & 0.00 & 0.00 & 0.00 & 0.00 & 0.00 & 0.00 & 0.00 & 0.36 & 0.00 \\
\hline 2 & .00 & .00 & .12 & .00 & .36 & .00 & .00 & .00 & .00 & .00 & .00 & .00 \\
\hline 3 & .00 & .00 & .00 & .00 & .72 & 2.04 & .00 & .00 & .00 & .00 & .12 & .00 \\
\hline 4 & .00 & .00 & .00 & .00 & 1.56 & 1.20 & .00 & .00 & .00 & .00 & .00 & .00 \\
\hline 5 & .00 & .00 & .00 & .00 & .12 & .00 & .00 & .00 & .00 & .00 & .00 & .00 \\
\hline 6 & .00 & .00 & .00 & .00 & .24 & .00 & .00 & .00 & .00 & .00 & .00 & .00 \\
\hline 7 & .00 & .00 & .00 & .00 & .84 & .12 & .00 & .00 & .00 & .84 & .36 & .00 \\
\hline 8 & .00 & 1.44 & .48 & .00 & 1.20 & .00 & .00 & .00 & .00 & .00 & .00 & .00 \\
\hline 9 & .00 & .84 & .84 & .00 & .00 & .00 & .00 & .00 & .00 & .48 & .00 & .00 \\
\hline 10 & .00 & 1.32 & .72 & .00 & .00 & .00 & .00 & .00 & .00 & .00 & .00 & .00 \\
\hline 11 & .00 & .00 & .12 & .00 & .00 & .00 & .00 & .00 & .00 & 1.20 & .24 & .00 \\
\hline 12 & .00 & .00 & .00 & .00 & .00 & .00 & .12 & .00 & .00 & .00 & .00 & .00 \\
\hline 13 & .00 & .00 & .00 & .00 & .00 & .00 & .12 & .00 & .00 & .00 & .00 & .00 \\
\hline 14 & .00 & .00 & .00 & .00 & .00 & .00 & .00 & .00 & .00 & .00 & .00 & .00 \\
\hline 15 & .00 & .00 & .00 & .00 & .00 & .00 & .00 & .00 & .00 & .00 & 1.08 & .00 \\
\hline 16 & .00 & .00 & .00 & .00 & .00 & .00 & .00 & .00 & .00 & .00 & .12 & .00 \\
\hline 17 & .00 & .84 & .00 & .00 & .00 & .36 & .00 & .00 & .00 & .00 & .60 & .00 \\
\hline 18 & .00 & .84 & .00 & .00 & .00 & .60 & .00 & .00 & .00 & .00 & .12 & .00 \\
\hline 19 & .00 & .00 & .00 & .00 & .00 & .84 & .00 & .00 & .00 & .00 & .00 & .00 \\
\hline 20 & .00 & .00 & .00 & .24 & .00 & .00 & .36 & .00 & .00 & .00 & .00 & .00 \\
\hline 21 & .00 & .00 & .00 & .00 & .00 & .60 & 1.20 & .00 & .00 & .00 & .00 & .00 \\
\hline 22 & .00 & .00 & .72 & .00 & .00 & .00 & .00 & .00 & .00 & 1.08 & .00 & .00 \\
\hline 23 & .00 & .00 & 1.20 & .00 & .00 & .60 & .00 & .00 & .00 & .00 & .00 & .00 \\
\hline 24 & .00 & .00 & .12 & .00 & .00 & .84 & .00 & .00 & .00 & .24 & .00 & .24 \\
\hline 25 & .00 & .00 & .00 & .00 & .00 & .00 & .00 & .00 & .00 & .12 & .00 & .00 \\
\hline 26 & .24 & .00 & .00 & .00 & .00 & .12 & .00 & .00 & .00 & .00 & .00 & .84 \\
\hline 27 & .00 & .00 & .00 & .84 & .00 & .00 & .00 & .00 & .00 & .00 & .00 & .00 \\
\hline 28 & .00 & .00 & .00 & .36 & .00 & .00 & .00 & .00 & .00 & .00 & .00 & .24 \\
\hline 29 & .00 & .00 & .00 & 1.44 & --- & .00 & .00 & .00 & .00 & .00 & .00 & 1.20 \\
\hline 30 & .00 & 2.40 & .24 & .00 & --- & .00 & .36 & .00 & .00 & .00 & .00 & 2.16 \\
\hline 31 & .00 & -- & .00 & .36 & --- & .00 & --- & .00 & $\cdots$ & .00 & .00 & --- \\
\hline Total & .24 & 7.68 & 4.56 & 3.24 & 5.04 & 7.32 & 2.16 & .00 & .00 & 3.96 & 3.00 & 4.68 \\
\hline
\end{tabular}

Total for water year $1983,41.88$ inches. 
Table 1. Estimated daily precipitation, East Fork and West Fork Sycamore Creek drainage basins, water years 1983-86-Continued

09510099 East Fork Sycamore Creek near Sunflower

Rainfall accumulated, in inches, water year October 1983 to September 1984

\begin{tabular}{|c|c|c|c|c|c|c|c|c|c|c|c|c|}
\hline Day & Oct. & Nov. & Dec. & Jan. & Feb. & Mar. & Apr. & May & June & July & Aug. & Sept. \\
\hline 1 & 1.56 & 0.00 & 0.24 & 0.00 & 0.00 & 0.00 & 0.00 & 0.00 & 0.00 & 0.00 & 0.00 & 0.12 \\
\hline 2 & .00 & .00 & .24 & .00 & .00 & .00 & .00 & .00 & .00 & .00 & .00 & .00 \\
\hline 3 & .12 & .00 & .12 & .00 & .00 & .00 & .00 & .00 & .00 & .00 & .00 & .00 \\
\hline 4 & .00 & .00 & .36 & .00 & .00 & .00 & .00 & .00 & .00 & .00 & .24 & .00 \\
\hline 5 & .00 & .00 & .00 & .12 & .00 & .00 & .84 & .00 & .00 & .00 & .00 & .00 \\
\hline 6 & .00 & .00 & .00 & .12 & .00 & .00 & .00 & .00 & .00 & .12 & .84 & .00 \\
\hline 7 & .00 & .00 & .00 & .00 & .00 & .00 & .00 & .00 & .00 & .00 & .00 & .00 \\
\hline 8 & .60 & .00 & .00 & .00 & .00 & .00 & .36 & .00 & .00 & .00 & .12 & .00 \\
\hline 9 & .00 & .00 & .00 & .00 & .00 & .00 & .00 & .00 & .00 & .00 & .24 & .00 \\
\hline 10 & .00 & .00 & .00 & .00 & .00 & .00 & .00 & .00 & .00 & .00 & .24 & .00 \\
\hline 11 & .00 & .00 & .00 & .00 & .00 & .00 & .00 & .00 & .00 & .00 & .00 & .00 \\
\hline 12 & .00 & .00 & .00 & .00 & .00 & .00 & .00 & .00 & .00 & .00 & 1.08 & .00 \\
\hline 13 & .00 & .00 & .00 & .00 & .00 & .00 & .00 & .00 & .00 & .12 & .00 & .00 \\
\hline 14 & .00 & .00 & .00 & .00 & .00 & .00 & .00 & .00 & .00 & .00 & .00 & .00 \\
\hline 15 & .00 & .00 & .00 & .00 & .00 & .00 & .00 & .00 & .00 & .00 & .00 & .00 \\
\hline 16 & .00 & .00 & .00 & .00 & .00 & .00 & .00 & .00 & .00 & .00 & .00 & .00 \\
\hline 17 & .00 & .00 & .00 & .00 & .00 & .00 & .00 & .00 & .00 & .12 & .12 & .00 \\
\hline 18 & .00 & .00 & .00 & .00 & .00 & .00 & .00 & .00 & .00 & .00 & .00 & .00 \\
\hline 19 & .00 & .00 & .00 & .00 & .00 & .00 & .00 & .00 & .00 & .96 & .36 & .00 \\
\hline 20 & .00 & .48 & .00 & .00 & .00 & .00 & .00 & .00 & .00 & .00 & .00 & .00 \\
\hline 21 & .00 & .96 & .00 & .00 & .00 & .00 & .00 & .00 & .00 & .12 & .00 & .00 \\
\hline 22 & .00 & .00 & .00 & .00 & .00 & .00 & .00 & .00 & .00 & .12 & .00 & .00 \\
\hline 23 & .00 & .00 & .00 & .00 & .00 & .00 & .00 & .00 & .00 & 1.68 & .00 & .00 \\
\hline 24 & .00 & .00 & .00 & .00 & .00 & .00 & .00 & .00 & .00 & .00 & .36 & .00 \\
\hline 25 & .00 & .36 & .72 & .00 & .00 & .00 & .00 & .00 & .00 & .00 & .00 & .72 \\
\hline 26 & .00 & .00 & .24 & .00 & .00 & .00 & .00 & .00 & .00 & .00 & .12 & 1.20 \\
\hline 27 & .00 & .00 & 1.68 & .00 & .00 & .00 & .24 & .00 & .60 & .00 & .00 & .00 \\
\hline 28 & .00 & .00 & .12 & .00 & .00 & .00 & .00 & .00 & .12 & .12 & .00 & .00 \\
\hline 29 & .00 & .00 & .00 & .00 & -- & .00 & .00 & .00 & .12 & .00 & .00 & .00 \\
\hline 30 & .00 & .00 & .00 & .00 & -- & .00 & .00 & .00 & .00 & .00 & .00 & .00 \\
\hline 31 & .00 & --- & .00 & .00 & -- & .00 & -- & .00 & --. & .00 & .00 & --- \\
\hline Total & 2.28 & 1.80 & 3.72 & .24 & .00 & .00 & 1.44 & .00 & .84 & 3.36 & 3.72 & 2.04 \\
\hline
\end{tabular}

Total for water year $1984,19.44$ inches. 
Table 1. Estimated daily precipitation, East Fork and West Fork Sycamore Creek drainage basins, water years 1983-86-Continued

09510099 East Fork Sycamore Creek near Sunfiower

Rainfall accumulated, In inches, water year October 1984 to September 1985

\begin{tabular}{|c|c|c|c|c|c|c|c|c|c|c|c|c|}
\hline Day & Oct. & Nov. & Dec. & Jan. & Feb. & Mar. & Apr. & May & June & July & Aug. & Sept. \\
\hline 1 & 0.00 & 0.00 & 0.00 & 0.00 & 0.00 & 0.00 & 0.00 & 0.00 & 0.00 & 0.00 & 0.00 & 0.00 \\
\hline 2 & .00 & .00 & .00 & .00 & .00 & .00 & .00 & .00 & .00 & .00 & .00 & .00 \\
\hline 3 & 2.52 & .00 & .00 & .00 & .48 & .00 & .00 & .00 & .00 & .00 & .00 & .00 \\
\hline 4 & .00 & .00 & .24 & .00 & 1.08 & .00 & .00 & .00 & .00 & .00 & .00 & .00 \\
\hline 5 & .00 & .00 & .12 & .00 & .00 & .00 & .00 & .00 & .00 & .00 & .00 & .00 \\
\hline 6 & .00 & .00 & .00 & .00 & .00 & .00 & .00 & .00 & .00 & .00 & .00 & .96 \\
\hline 7 & .00 & .00 & .00 & .24 & .00 & .00 & .00 & .00 & .00 & .00 & .00 & .12 \\
\hline 8 & .00 & 0.00 & .48 & 1.08 & .00 & .00 & .00 & .00 & .00 & .00 & .00 & .00 \\
\hline 9 & .00 & .00 & .12 & .12 & .00 & .00 & .00 & .00 & .00 & .00 & .00 & .00 \\
\hline 10 & .00 & .00 & .36 & .00 & .00 & .00 & .00 & .00 & .00 & .00 & .00 & .00 \\
\hline 11 & .00 & .00 & .72 & .00 & .00 & .60 & .00 & .00 & .00 & .00 & .00 & .72 \\
\hline 12 & .48 & .00 & .60 & .00 & .00 & .12 & .00 & .00 & .00 & .00 & .00 & .00 \\
\hline 13 & .00 & .00 & .48 & .00 & .00 & .00 & .00 & .00 & .00 & .00 & .00 & .00 \\
\hline 14 & .00 & .00 & .24 & .00 & .00 & .12 & .00 & .00 & .00 & 1.80 & .00 & .00 \\
\hline 15 & .48 & .00 & .12 & .00 & .00 & .36 & .00 & .00 & .00 & .72 & .00 & .00 \\
\hline 16 & .24 & .00 & .12 & .00 & .00 & .12 & .00 & .00 & .00 & .00 & .00 & .00 \\
\hline 17 & .00 & .00 & .00 & .00 & .00 & .00 & .00 & .00 & .00 & .00 & .00 & .00 \\
\hline 18 & .00 & .12 & .00 & .00 & .00 & .00 & .12 & .00 & .00 & .72 & .00 & 1.08 \\
\hline 19 & .00 & .00 & .00 & .00 & .00 & .00 & .00 & .00 & .00 & .00 & .00 & .00 \\
\hline 20 & .00 & .00 & .24 & .00 & .12 & .00 & .00 & .00 & .00 & .00 & .36 & .00 \\
\hline 21 & .00 & .00 & .00 & .00 & .84 & .00 & .00 & .00 & .00 & .24 & .00 & .00 \\
\hline 22 & .00 & .12 & .00 & .00 & .12 & .00 & .12 & .00 & .00 & .00 & .00 & .00 \\
\hline 23 & .00 & .84 & .00 & .36 & .00 & .00 & .00 & .00 & .00 & .00 & .00 & .00 \\
\hline 24 & .00 & .36 & .00 & .12 & .00 & .00 & .00 & .00 & .00 & .00 & .00 & .00 \\
\hline 25 & .00 & 1.80 & .00 & .12 & .00 & .12 & .00 & .00 & .00 & .00 & .00 & .00 \\
\hline 26 & .00 & .00 & .00 & .96 & .00 & .00 & .00 & .00 & .00 & .00 & .00 & .00 \\
\hline 27 & .00 & .00 & 2.52 & .72 & .00 & .00 & .48 & .00 & .00 & .00 & .00 & .00 \\
\hline 28 & .00 & .00 & .36 & .00 & .00 & .60 & .12 & .00 & .00 & .00 & .00 & .00 \\
\hline 29 & .00 & .00 & .00 & .12 & --- & .24 & .00 & .00 & .00 & .00 & .00 & .00 \\
\hline 30 & .00 & .00 & .00 & .12 & --- & .00 & .00 & .00 & .00 & .00 & .00 & .00 \\
\hline 31 & .00 & -- & .00 & .24 & -- & .00 & $\cdots$ & .00 & -- & .00 & .00 & --- \\
\hline Total & 3.72 & 3.24 & 6.72 & 4.20 & 2.64 & 2.28 & 1.44 & .00 & .00 & 3.48 & .36 & 2.88 \\
\hline
\end{tabular}

Total for water year 1985, 30.96 inches. 
Table 1. Estimated daily precipitation, East Fork and West Fork Sycamore Creek drainage basins, water years 1983-86-Continued

\begin{tabular}{|c|c|c|c|c|c|c|c|c|}
\hline \multicolumn{9}{|c|}{$\begin{array}{l}09510099 \text { East Fork Sycamore Creek near Sunflower } \\
\text { Ralnfall accumulated, In Inches, October } 1985 \text { to May } 1986\end{array}$} \\
\hline Day & Oct. & Nov. & Dec. & Jan. & Feb. & Mar. & Apr. & May \\
\hline 1 & 0.00 & 0.00 & 0.00 & 0.00 & 0.00 & 0.00 & 0.00 & 0.00 \\
\hline 2 & .00 & .00 & .00 & .00 & .00 & .00 & .12 & .00 \\
\hline 3 & .00 & .00 & .00 & .00 & .00 & .00 & .00 & .00 \\
\hline 4 & .00 & .00 & .00 & .00 & .00 & .00 & .00 & .00 \\
\hline 5 & .00 & .00 & .00 & .00 & .00 & .00 & .00 & .00 \\
\hline 6 & .00 & .00 & .00 & .00 & .00 & .00 & .00 & .00 \\
\hline 7 & .00 & .00 & .00 & .00 & .00 & .00 & .00 & .00 \\
\hline 8 & .00 & .00 & .00 & .00 & .00 & .00 & .00 & .00 \\
\hline 9 & .00 & .00 & .00 & .00 & .84 & .00 & .00 & .00 \\
\hline 10 & .60 & .00 & .00 & .00 & .72 & .36 & .00 & .00 \\
\hline 11 & .00 & .00 & .00 & .00 & .00 & 1.20 & .00 & .00 \\
\hline 12 & .00 & 1.44 & .24 & .00 & .00 & .48 & .00 & .00 \\
\hline 13 & .00 & .00 & .00 & .00 & .00 & .12 & .00 & .00 \\
\hline 14 & .00 & .00 & .00 & .00 & .00 & .48 & .00 & .00 \\
\hline 15 & .60 & .00 & .72 & .00 & 1.56 & .12 & .00 & .00 \\
\hline 16 & .00 & .00 & .00 & .00 & .36 & .96 & .00 & .00 \\
\hline 17 & .96 & .00 & .00 & .00 & .00 & .12 & .00 & .00 \\
\hline 18 & .00 & .00 & .00 & .00 & .00 & .72 & .00 & .00 \\
\hline 19 & .00 & .00 & .00 & .00 & .00 & .00 & .00 & .00 \\
\hline 20 & .00 & .00 & .00 & .00 & .00 & .00 & .00 & .00 \\
\hline 21 & .00 & .00 & .00 & .00 & .00 & .00 & .00 & .00 \\
\hline 22 & .00 & .00 & .00 & .00 & .00 & .00 & .00 & .00 \\
\hline 23 & .00 & .00 & .00 & .00 & .00 & .00 & .00 & .00 \\
\hline 24 & .00 & .00 & .00 & .00 & .00 & .00 & .00 & .00 \\
\hline 25 & .00 & 1.08 & .00 & .00 & .00 & .00 & .00 & .00 \\
\hline 26 & .00 & 1.32 & .00 & .00 & .00 & .00 & .00 & .00 \\
\hline 27 & .00 & .00 & .00 & .00 & .00 & .00 & .00 & .00 \\
\hline 28 & .00 & .00 & .00 & .00 & .00 & .00 & .00 & .00 \\
\hline 29 & .00 & .00 & .00 & .00 & --- & .00 & .00 & .00 \\
\hline 30 & .00 & 1.32 & .00 & .240 & --- & .00 & .00 & .00 \\
\hline 31 & .00 & --- & .00 & .00 & --- & .00 & -- & .36 \\
\hline Total & 2.16 & 5.16 & .96 & .24 & 3.48 & 4.56 & .12 & .36 \\
\hline
\end{tabular}

Total for October 1985 through May 1986, 17.04 inches. 
Table 1. Estimated daily precipitation, East Fork and West Fork Sycamore Creek drainage basins, water years 1983-86-Continued

\begin{tabular}{|c|c|c|c|c|c|c|c|c|c|c|c|c|}
\hline \multicolumn{13}{|c|}{$\begin{array}{l}09510069 \text { West Fork Sycamore Creek above McFarland Canyon near Sunflower } \\
\text { Rainfall accumulated, in inches, water year October } 1982 \text { to September } 1983\end{array}$} \\
\hline Day & Oct. & Nov. & Dec. & Jan. & Feb. & Mar. & Apr. & May & June & July & Aug. & Sept. \\
\hline 1 & 0.00 & 0.00 & 0.48 & 0.12 & 0.00 & 0.00 & 0.00 & 0.00 & 0.00 & 0.00 & 0.12 & 0.00 \\
\hline 2 & .00 & .00 & .48 & .12 & .12 & .00 & .00 & .00 & .00 & .00 & .00 & .00 \\
\hline 3 & .00 & .00 & .00 & .00 & .84 & 2.40 & .00 & .00 & .00 & .00 & .00 & .00 \\
\hline 4 & .00 & .00 & .00 & .00 & 1.68 & 1.80 & .00 & .00 & .00 & .00 & .00 & .00 \\
\hline 5 & .00 & .00 & .00 & .00 & .12 & .00 & .00 & .00 & .00 & .00 & .00 & .00 \\
\hline 6 & .00 & .00 & .00 & .00 & .12 & .00 & .00 & .00 & .00 & .00 & .24 & .00 \\
\hline 7 & .00 & .00 & .00 & .00 & .72 & .00 & .00 & .00 & .00 & .00 & .12 & .00 \\
\hline 8 & .00 & 1.00 & .36 & .00 & 1.32 & .00 & .00 & .00 & .00 & .00 & .00 & .00 \\
\hline 9 & .00 & 1.52 & .84 & .00 & .00 & .00 & .00 & .00 & .00 & .00 & .00 & .00 \\
\hline 10 & .00 & 1.56 & .84 & .00 & .00 & .00 & .00 & .00 & .00 & .00 & .00 & .00 \\
\hline 11 & .00 & .00 & .00 & .00 & .00 & .00 & .00 & .00 & .00 & .00 & .36 & .00 \\
\hline 12 & .00 & .00 & .00 & .00 & .00 & .00 & .00 & .00 & .00 & .00 & .00 & .00 \\
\hline 13 & .00 & .00 & .00 & .00 & .00 & .00 & .12 & .00 & .00 & .00 & .00 & .00 \\
\hline 14 & .00 & .00 & .00 & .00 & .00 & .00 & .00 & .00 & .00 & .00 & .00 & .00 \\
\hline 15 & .00 & .00 & .00 & .00 & .00 & .00 & .00 & .00 & .00 & .00 & 1.08 & .00 \\
\hline 16 & .00 & .00 & .00 & .00 & .00 & .00 & .00 & .00 & .00 & .00 & .12 & .00 \\
\hline 17 & .00 & 1.44 & .00 & .00 & .00 & .48 & .00 & .00 & .00 & .00 & .84 & .00 \\
\hline 18 & .00 & .72 & .00 & .00 & .00 & .84 & .00 & .00 & .00 & .00 & .00 & .00 \\
\hline 19 & .00 & .00 & .00 & .12 & .00 & .84 & .00 & .00 & .00 & .00 & .00 & .00 \\
\hline 20 & .00 & .00 & .00 & .00 & .00 & .00 & .24 & .00 & .00 & .00 & .00 & .12 \\
\hline 21 & .00 & .00 & .00 & .00 & .00 & .72 & .96 & .00 & .00 & .72 & .00 & .00 \\
\hline 22 & .00 & .00 & .84 & .00 & .00 & .12 & .00 & .00 & .00 & .00 & .00 & .00 \\
\hline 23 & .00 & .00 & 1.56 & .00 & .00 & .72 & .00 & .00 & .00 & .00 & .00 & .00 \\
\hline 24 & .00 & .00 & .00 & .00 & .00 & .96 & .00 & .00 & .00 & .24 & .00 & .24 \\
\hline 25 & .00 & .00 & .00 & .00 & .00 & .00 & .00 & .00 & .00 & .00 & .00 & .00 \\
\hline 26 & .00 & .00 & .00 & .00 & .00 & .12 & .00 & .00 & .00 & .00 & .00 & 1.68 \\
\hline 27 & .00 & .00 & .00 & 1.20 & .00 & .00 & .00 & .00 & .00 & .00 & .00 & .00 \\
\hline 28 & .00 & .00 & .12 & .60 & .00 & .00 & .00 & .00 & .00 & .00 & .00 & .00 \\
\hline 29 & .00 & .12 & .12 & 1.80 & --- & .00 & .00 & .00 & .00 & .00 & .00 & 1.32 \\
\hline 30 & .00 & 4.20 & .00 & .00 & --- & .00 & .24 & .00 & .00 & .00 & .00 & 2.64 \\
\hline 31 & .00 & --- & .00 & .36 & --- & .00 & --- & .00 & --- & .36 & .00 & -- \\
\hline Total & 0.00 & 10.56 & 5.64 & 4.32 & 4.92 & 9.00 & 1.68 & .00 & .00 & 1.32 & 2.88 & 6.00 \\
\hline
\end{tabular}

Total for water year $1983,46.32$ inches. 
Table 1. Estimated daily precipitation, East Fork and West Fork Sycamore Creek drainage basins, water years 1983-86-Continued

\begin{tabular}{|c|c|c|c|c|c|c|c|c|c|c|c|c|}
\hline \multicolumn{13}{|c|}{$\begin{array}{l}09510069 \text { West Fork Sycamore Creek above McFarland Canyon near Sunflower } \\
\text { Rainfall accumulated, in inches, water year October } 1983 \text { to September } 1984\end{array}$} \\
\hline Day & Oct. & Nov. & Dec. & Jan. & Feb. & Mar. & Apr. & May & June & July & Aug. & Sept. \\
\hline 1 & 1.80 & 0.00 & 0.12 & 0.00 & 0.00 & 0.00 & 0.00 & 0.00 & 0.00 & 0.00 & 0.00 & 0.48 \\
\hline 2 & .12 & .00 & .72 & .00 & .00 & .00 & .00 & .00 & .00 & .12 & .00 & .00 \\
\hline 3 & .12 & .00 & .12 & .00 & .00 & .00 & .00 & .00 & .00 & .00 & .00 & .00 \\
\hline 4 & .00 & .00 & .48 & .00 & .00 & .00 & .00 & .00 & .00 & .00 & .24 & .00 \\
\hline 5 & .00 & .00 & .12 & .12 & .00 & .00 & .84 & .00 & .00 & .00 & .00 & .00 \\
\hline 6 & .00 & .00 & .00 & .12 & .00 & .00 & .00 & .00 & .00 & .00 & .84 & .00 \\
\hline 7 & .00 & .00 & .00 & .00 & .00 & .00 & .00 & .00 & .00 & .12 & .00 & .00 \\
\hline 8 & .48 & .00 & .00 & .00 & .00 & .00 & .36 & .00 & .00 & .00 & .24 & .00 \\
\hline 9 & .00 & .00 & .00 & .00 & .00 & .00 & .00 & .00 & .00 & .00 & .48 & .12 \\
\hline 10 & .00 & .00 & .00 & .00 & .00 & .00 & .00 & .00 & .00 & .00 & .24 & .36 \\
\hline 11 & .00 & .00 & .00 & .00 & .00 & .00 & .00 & .00 & .00 & .00 & .00 & .24 \\
\hline 12 & .00 & .00 & .00 & .00 & .00 & .00 & .00 & .00 & .00 & .12 & .10 & .00 \\
\hline 13 & .00 & .00 & .00 & .00 & .00 & .00 & .00 & .00 & .00 & .12 & .00 & .00 \\
\hline 14 & .00 & .00 & .00 & .00 & .00 & .00 & .00 & .00 & .12 & .00 & .00 & .00 \\
\hline 15 & .00 & .00 & .00 & .00 & .00 & .00 & .00 & .00 & .00 & .00 & .00 & .00 \\
\hline 16 & .00 & .00 & .00 & .00 & .00 & .00 & .00 & .00 & .00 & .00 & .00 & .00 \\
\hline 17 & .00 & .00 & .00 & .00 & .00 & .00 & .00 & .00 & .00 & .00 & .24 & .12 \\
\hline 18 & .00 & .00 & .00 & .00 & .00 & .00 & .00 & .00 & .00 & .00 & .00 & .00 \\
\hline 19 & .00 & .00 & .00 & .12 & .00 & .00 & .00 & .00 & .00 & .48 & .24 & .00 \\
\hline 20 & .00 & .36 & .00 & .00 & .00 & .00 & .00 & .00 & .00 & .12 & .00 & .00 \\
\hline 21 & .00 & 1.20 & .00 & .00 & .00 & .00 & .00 & .00 & .00 & .24 & .00 & .00 \\
\hline 22 & .00 & .36 & .00 & .00 & .00 & .00 & .00 & .00 & .12 & .12 & .00 & .00 \\
\hline 23 & .00 & .00 & .00 & .00 & .00 & .00 & .00 & .00 & .00 & .36 & .24 & .00 \\
\hline 24 & .00 & .00 & .00 & .24 & .00 & .00 & .00 & .00 & .00 & .00 & .00 & .00 \\
\hline 25 & .00 & .36 & .72 & .00 & .00 & .00 & .00 & .00 & .00 & .00 & .00 & .84 \\
\hline 26 & .00 & .00 & .24 & .00 & .00 & .00 & .00 & .00 & .36 & .00 & .60 & .20 \\
\hline 27 & .00 & .00 & 1.56 & .00 & .00 & .00 & .00 & .00 & .00 & .00 & .00 & .00 \\
\hline 28 & .00 & .00 & .00 & .00 & .00 & .00 & .00 & .00 & .12 & .00 & .12 & .12 \\
\hline 29 & .00 & .00 & .12 & .00 & --- & .00 & .00 & .00 & .24 & .00 & .00 & .00 \\
\hline 30 & .00 & .00 & .00 & .00 & --- & .00 & .00 & .00 & .00 & .00 & .00 & .00 \\
\hline 31 & .00 & --- & .00 & .00 & --- & .00 & --- & .00 & -.- & .00 & .00 & --- \\
\hline Total & 2.52 & 2.28 & 4.20 & .60 & .00 & .00 & 1.20 & .00 & .84 & 1.92 & 4.58 & .48 \\
\hline
\end{tabular}

Total for water year 1984, 21.62 inches. 
Table 1. Estimated daily precipitation, East Fork and West Fork Sycamore Creek drainage basins, water years 1983-86-Continued

\begin{tabular}{|c|c|c|c|c|c|c|c|c|c|c|c|c|}
\hline \multicolumn{13}{|c|}{$\begin{array}{l}09510069 \text { West Fork Sycamore Creek above McFarland Canyon near Sunfiower } \\
\text { Rainfall accumulated, in inches, water year October } 1984 \text { to September } 1985\end{array}$} \\
\hline Day & Oct. & Nov. & Dec. & Jan. & Feb. & Mar. & Apr. & May & June & July & Aug. & Sept. \\
\hline 1 & 0.00 & 0.00 & 0.00 & 0.00 & 0.00 & 0.00 & 0.00 & 0.00 & 0.00 & 0.00 & 0.00 & 0.00 \\
\hline 2 & .00 & .00 & .00 & .00 & .00 & .00 & .00 & .00 & .00 & .00 & .00 & .00 \\
\hline 3 & 3.96 & .00 & .00 & .00 & .48 & .00 & .00 & .00 & .00 & .00 & .00 & .00 \\
\hline 4 & .00 & .00 & .24 & .00 & 1.20 & .12 & .00 & .00 & .00 & .00 & .00 & .00 \\
\hline 5 & .00 & .00 & .12 & .00 & .00 & .00 & .00 & .00 & .00 & .00 & .00 & .00 \\
\hline 6 & .12 & .00 & .00 & .00 & .00 & .00 & .00 & .00 & .00 & .00 & .00 & .48 \\
\hline 7 & .00 & .00 & .00 & .00 & .00 & .00 & .00 & .00 & .00 & .00 & .00 & .12 \\
\hline 8 & .00 & .00 & .48 & .24 & .00 & .00 & .00 & .00 & .00 & .00 & .00 & .00 \\
\hline 9 & .00 & .00 & .12 & 1.08 & .00 & .00 & .00 & .00 & .00 & .00 & .00 & .00 \\
\hline 10 & .00 & .00 & .36 & .12 & .00 & .00 & .00 & .00 & .00 & .00 & .00 & .00 \\
\hline 11 & .00 & .00 & .84 & .00 & .00 & .48 & .00 & .00 & .00 & .00 & .00 & .72 \\
\hline 12 & .84 & .00 & .72 & .00 & .00 & .36 & .00 & .00 & .00 & .00 & .00 & .00 \\
\hline 13 & .00 & .00 & .60 & .00 & .00 & .00 & .00 & .00 & .00 & .00 & .00 & .00 \\
\hline 14 & .00 & .00 & .24 & .00 & .00 & .00 & .00 & .00 & .00 & .60 & .00 & .00 \\
\hline 15 & 1.08 & .00 & .12 & .00 & .00 & .24 & .00 & .00 & .00 & .48 & .00 & .00 \\
\hline 16 & .00 & .00 & .12 & .00 & .00 & .12 & .00 & .00 & .00 & .12 & .00 & .00 \\
\hline 17 & .36 & .00 & .00 & .00 & .00 & .12 & .00 & .00 & .00 & .00 & .00 & .00 \\
\hline 18 & .00 & .12 & .00 & .00 & .00 & .00 & .12 & .00 & .00 & 1.44 & .00 & 1.08 \\
\hline 19 & .00 & .00 & .00 & .00 & .00 & .00 & .00 & .00 & .00 & .00 & .00 & .00 \\
\hline 20 & .00 & .00 & .24 & .00 & .00 & .12 & .12 & .00 & .00 & .12 & .48 & .00 \\
\hline 21 & .00 & .00 & .24 & .00 & .84 & .00 & .60 & .00 & .00 & .48 & .00 & .00 \\
\hline 22 & .00 & .12 & .00 & .00 & .24 & .00 & .12 & .00 & .00 & .00 & .00 & .00 \\
\hline 23 & .00 & .96 & .00 & .36 & .00 & .00 & .00 & .00 & .00 & .00 & .00 & .00 \\
\hline 24 & .00 & .36 & .00 & .00 & .00 & .00 & .00 & .00 & .00 & .00 & .00 & .00 \\
\hline 25 & .12 & 2.16 & .00 & .12 & .00 & .00 & .00 & .00 & .00 & .00 & .12 & .00 \\
\hline 26 & .00 & .00 & .00 & 1.08 & .00 & .00 & .00 & .00 & .00 & .00 & .00 & .00 \\
\hline 27 & .00 & .00 & 2.64 & .72 & .00 & .00 & .48 & .00 & .00 & .00 & .00 & .00 \\
\hline 28 & .00 & .00 & .84 & .00 & .00 & .96 & .12 & .00 & .00 & .00 & .00 & .00 \\
\hline 29 & .00 & .00 & .00 & .12 & --- & .72 & .00 & .00 & .00 & .00 & .00 & .00 \\
\hline 30 & .00 & .00 & .00 & .00 & $\cdots$ & .12 & .00 & .00 & .00 & .36 & .00 & .00 \\
\hline 31 & .00 & --- & .00 & .24 & --- & .00 & --- & .00 & --- & .00 & .00 & --. \\
\hline Total & 6.48 & 3.72 & 7.92 & 4.08 & 2.76 & 3.36 & 1.56 & .00 & .00 & 3.60 & .60 & 2.40 \\
\hline
\end{tabular}

Total for water year 1985, 36.48inches. 
Table 1. Estimated daily precipitation, East Fork and West Fork Sycamore Creek drainage basins, water years 1983-86-Continued

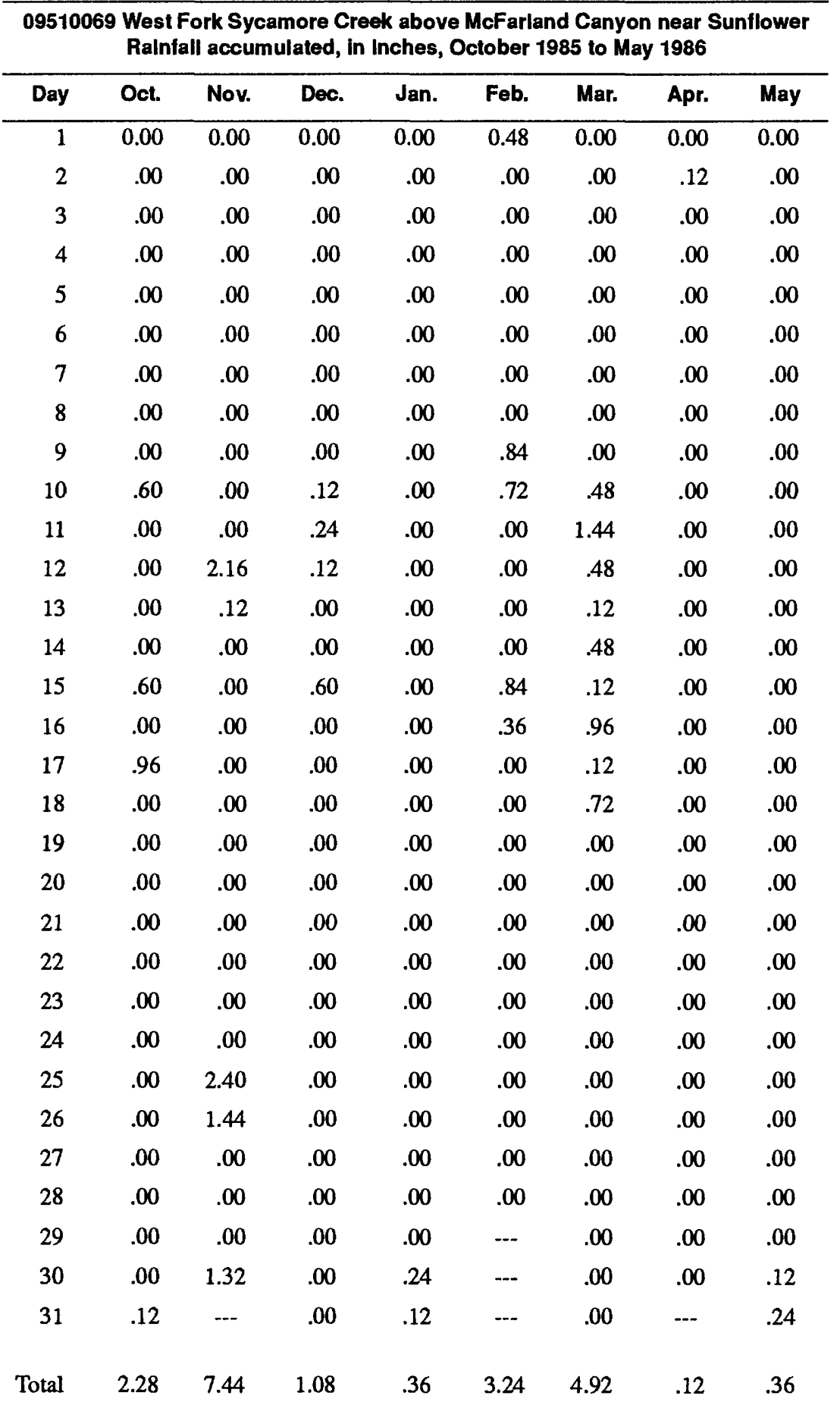

Total for October 1985 through May 1986, 19.80 inches. 


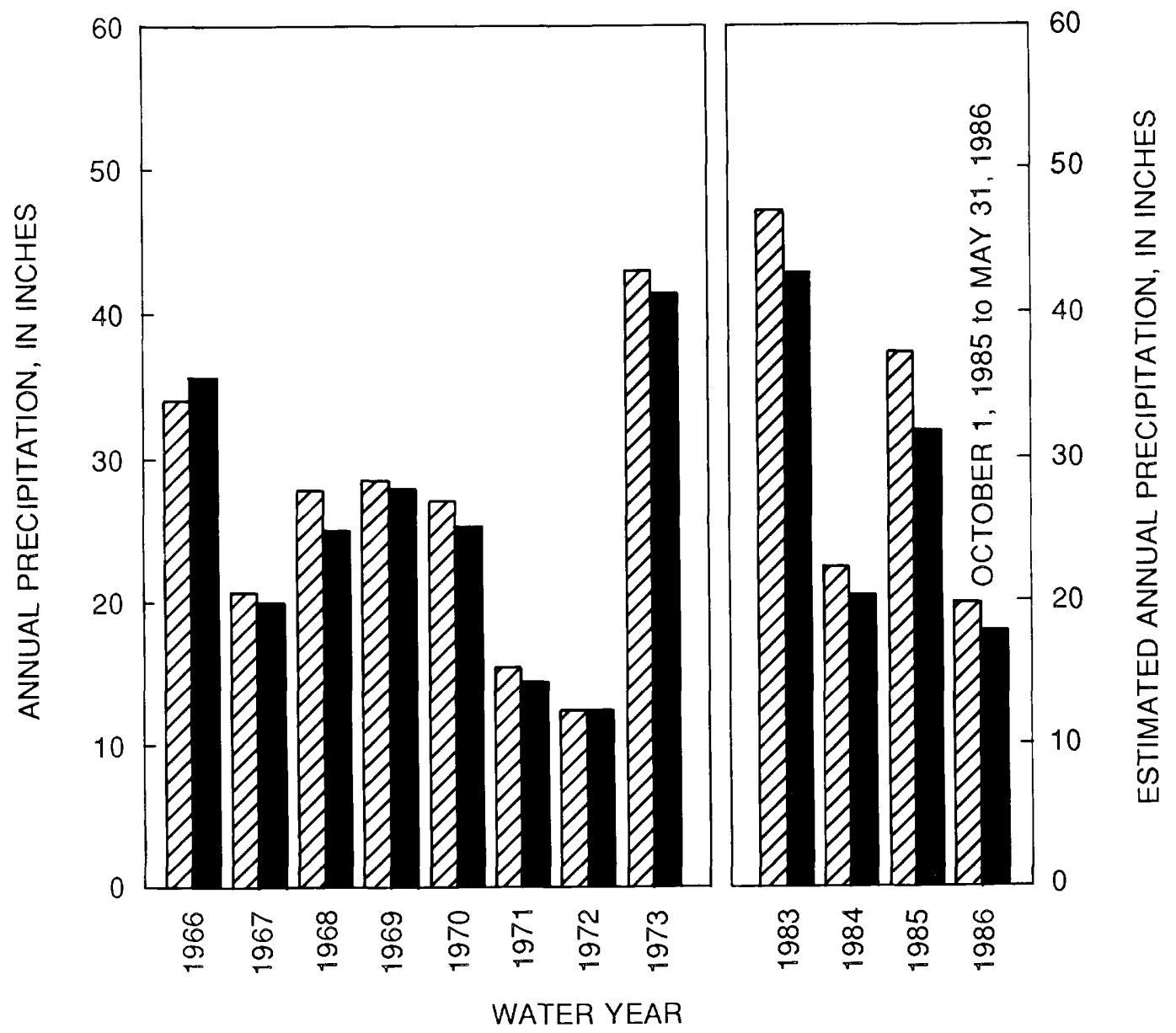

EXPLANATION

WEST FORK DRAINAGE BASIN
EAST FORK DRAINAGE BASIN

Figure 2. Annual precipitation for water years 1966-73 and estimated annual precipitation for water years 1983-86, East Fork and West Fork Sycamore Creek drainage basins.

annual precipitation may have resulted in runoff differences between the basins that were not experienced during the preburn period and were not the result of the burning of the vegetation. Because the basins are similar and there is no statistically significant difference of annual precipitation between the basins, the large amount of precipitation for 1983 does not appear to affect the analysis.

\section{Effects of Burning on Streamflow}

The effects of the burn on streamflow of the East Fork basin were analyzed using several 


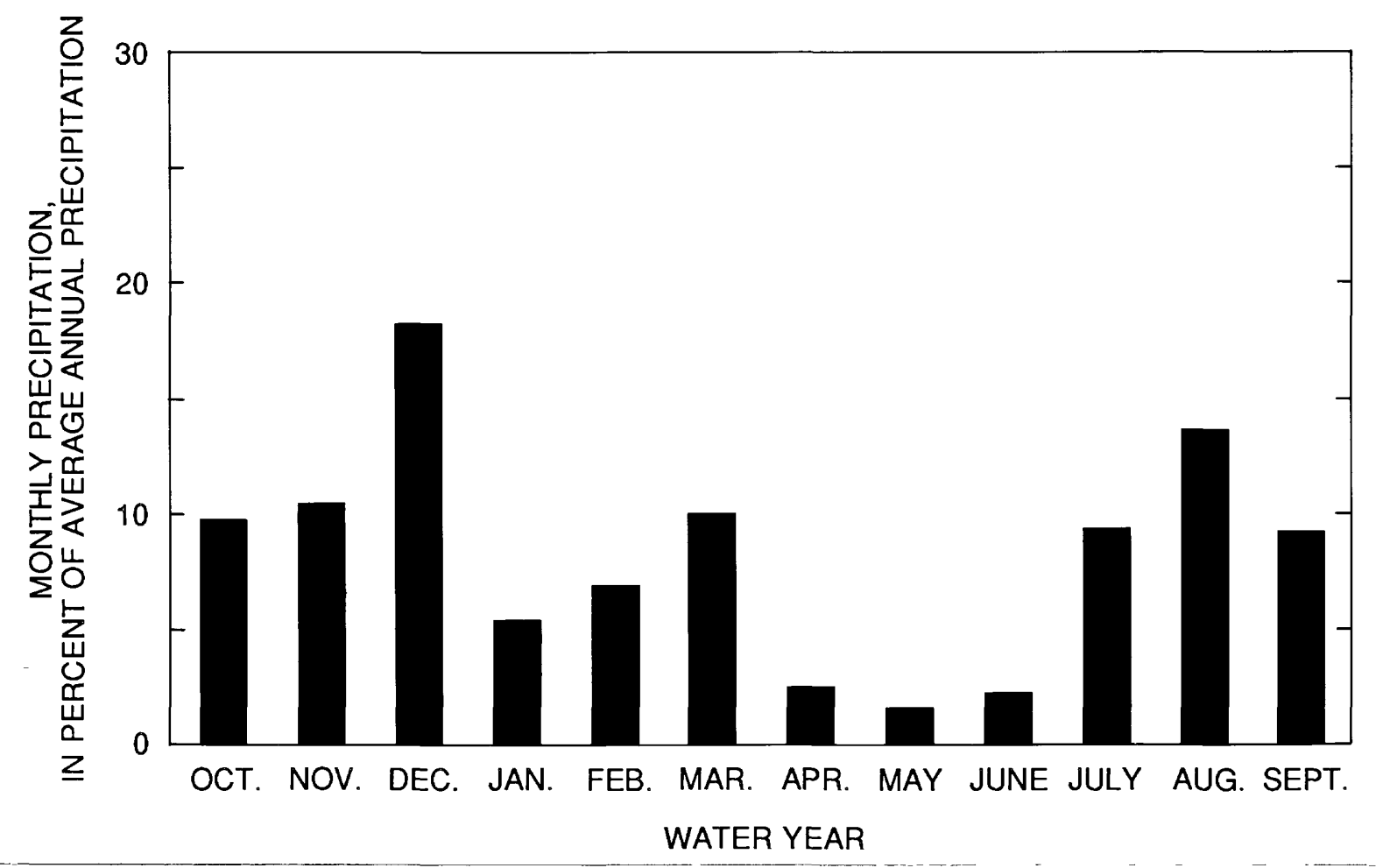

Figure 3. Monthly precipitation as a percentage of average annual precipitation, East Fork and West Fork Sycamore Creek drainage basins, water years 1966-73.

statistical techniques. Annual discharge, storm runoff, and instantaneous peak discharge were variables selected for the quantitative analysis. Flow-duration curves of daily discharge for the preburn and postburn periods were used for a qualitative analysis of the effects of the burn on streamflow. Several statistical techniques and streamflow variables were used because the reliability of the detection-of-change procedure depends on the size of the sample, the variability of the sample, the type of variable used, and the particular statistical technique used to detect the change, if any, of the streamflow variable. Discharge data at three springs also were analyzed to determine if changes occurred as a result of the burn in the East Fork basin.

\section{Annual Discharge}

Annual discharge measured at streamflowgaging stations during the postburn period was compared with annual discharge measured during the preburn period to determine if changes in streamflow were caused by the burn. The comparison was made using the preburn relation between discharge in the East Fork and West Fork basins defined by Hjalmarson (1975, p.57) as follows:

$$
\log Y=-0.49+1.13(\log X)
$$

where

$$
\begin{aligned}
Y= & \text { annual discharge, in acre-feet, East } \\
& \text { Fork drainage basin, and } \\
X= & \text { annual discharge, in acre-feet, West } \\
& \text { Fork drainage basin. }
\end{aligned}
$$

Equation 1 has a coefficient of determination, $R^{2}$ of 0.99 , indicating that nearly all the measured variation in annual discharge from East Fork basin was explained by the annual discharge from West Fork basin. The standard error of the regression equation is 0.0775 in log units. The mean of the logarithms of the $\mathrm{X}$ values (West Fork) is 2.433, and the mean of the logarithms of the $Y$ values (East 
Fork) is 2.260 . Discharge data were transformed to base 10 logarithms to ensure an equal variance about the regression line.

An optimal means to evaluate if a change of annual discharge occurred as a result of the burn would be to define the postburn relation between the two basins in annual discharge and compare that relation with the preburn relation using covariance analysis. A visual examination, however, indicates that the postburn annual streamflow is nonstationary. The annual streamflow for 1983 and 1984 plot to the left of equation 1 (fig. 4), and the annual streamflow for 1985 and the streamflow for part of 1986 plot to the right of equation 1 . Although not conclusive because of the small size of sample, the relation of annual streamflow between the basins appears to have changed with time since the burn. The burned chaparral vegetation was regrowing following the burn, and the amount of evapotranspiration from the East Fork basin may have returned to preburn levels. Apparently, the regenerative capacity of chaparral is supported by stored reserves of nutrients in the root crown and an extensive root system (Davis and Pase, 1977, p. 174). Thus, covariance methods were considered invalid, and postburn discharge for each year was analyzed separately.

Annual postburn discharge for the East Fork basin was computed using the preburn-streamflow relation (eq.1) and the corresponding annual discharge for the West Fork basin (table 2). The computed annual discharge was compared with the measured discharge. Only the streamflow for 1984 showed an apparent increase as a result of the burn at the 95-percent probability level (table 2). The measured discharge for 1983 showed an apparent increase only at the 65 -percent probability level (table 2). The measured discharge for the East Fork basin for 1985 and part of 1986 was less than the computed discharge, indicating that the streamflow quantities returned to preburn levels or, as shown by the discharge for 1985, may have been less than preburn levels. The changing relation is considered related to the regrowth of the chaparral vegetation.

The large annual discharge for the East Fork basin for 1984 appears to be the result of the burn. If the annual discharge totals are considered to be independent, then the probability of total annual discharge not exceeding the 95-percent probability level during the 4 postburn years is $(0.95)^{4}=0.81$. It follows that the probability of total annual discharge exceeding the limit of the 95-percent prediction interval (fig. 4) during the postburn period was only about 0.19 . Thus, annual discharge appears to have increased 49 percent for 1984 as a result of the vegetation burn and returned to preburn levels in 1985 and 1986.

The less-than-predicted discharge on the East Fork basin for 1985 may have been the result of storms bypassing the basin. Another possible cause for the difference may have been the rapid growth of chaparral attached to a large preburn root system that consumed larger-than-normal amounts of moisture. Also, part or all of the difference could be the result of the potential error in equation 1, which does not consider hydrologic variables other than discharge for the two basins.

Table 2. Measured and computed annual discharge, East Fork and West Fork Sycamore Creek drainage basins, water years $1983-86$

[Dashes indicate no data]

\begin{tabular}{|c|c|c|c|c|}
\hline & \multicolumn{4}{|c|}{ Discharge, in acre-feet, for indicated water year } \\
\hline & 1983 & 1984 & 1985 & $1986^{1}$ \\
\hline 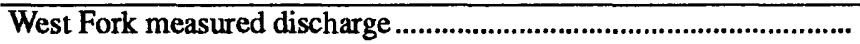 & 2,450 & 294 & 1,110 & 271 \\
\hline East Fork measured discharge & 2,410 & 298 & 624 & 178 \\
\hline 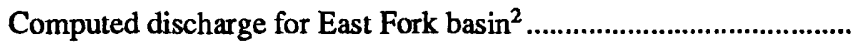 & 2,186 & 199 & 894 & 182 \\
\hline Probability level of an increase in annual discharge, in percent ........ & 65 & 95 & $-\cdots$ & $-\cdots$ \\
\hline
\end{tabular}

\footnotetext{
${ }^{1}$ Partial year, October 1 through May $31,1986$.

${ }^{2}$ Computed from equation 1 .
} 
18

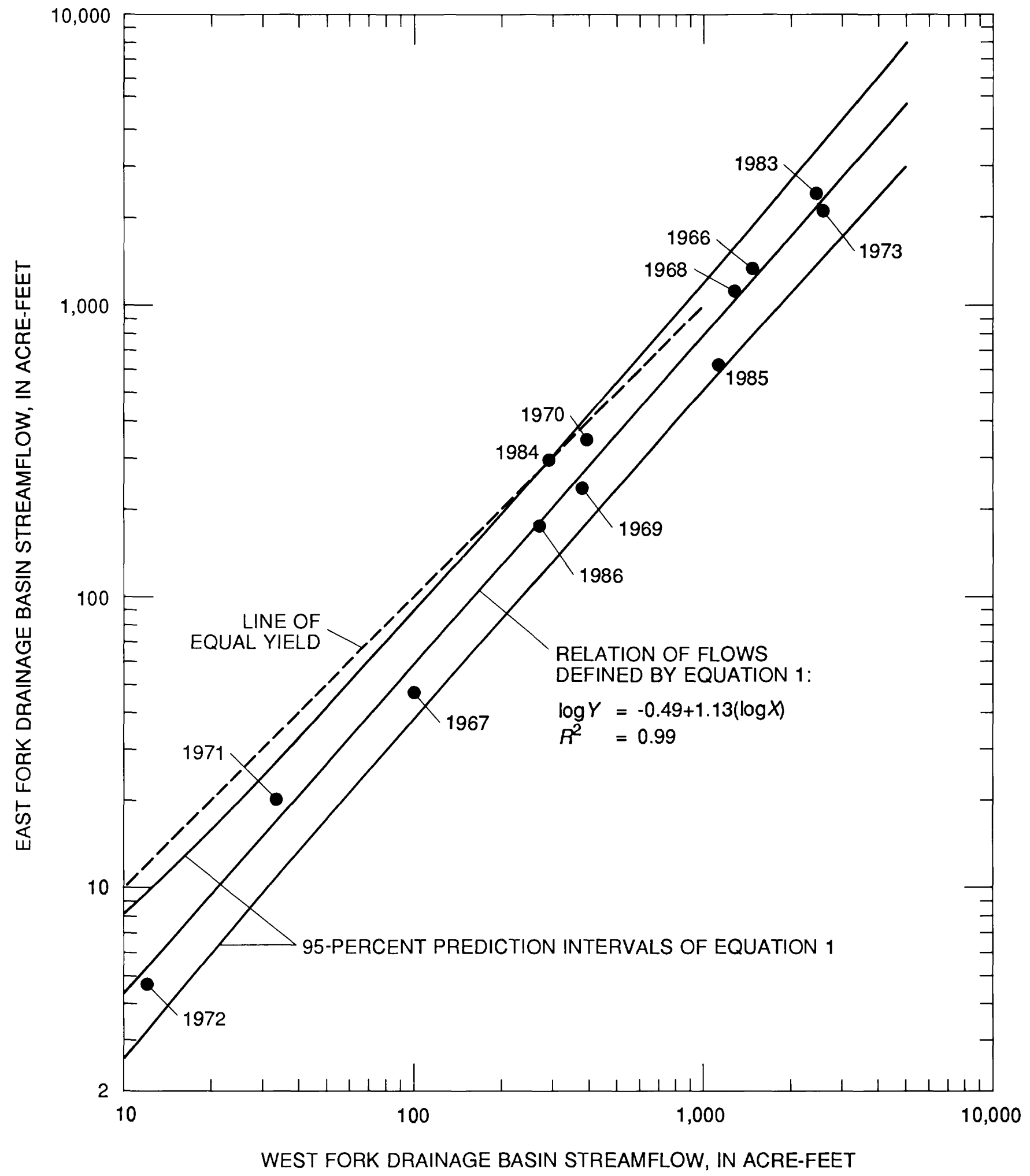

Figure 4. Annual discharge in East Fork Sycamore Creek drainage basin and annual discharge in West Fork Sycamore Creek drainage basin, water years 1966-73 and 1983-86. 
Investigations for similar studies of six drainage basins in Arizona indicated an average annual increase in streamflow of 28 percent (0.33 in.) at sites where open brush was removed and rainfall was low. Streamflow increased to 300 percent (more than 5 in.) at sites where dense brush was removed and rainfall was high (Hibbert and others, 1975, p. 452).

Annual discharge did not increase in 1983 (95-percent probability level) but appeared to increase in 1984 as a result of the burned chaparral. This disparity may be related to the large difference in streamflow between 1983 and 1984. Streamflow in 1984 was only about 10 percent of the streamflow in 1983 , which was the highest of the study period. Effects of the burn may be nonlinear and smaller for wet years than for dry years. Possible effects of the burn may include the reduction of precipitation intercepted in the vegetation canopy, the rapid regrowth of the chaparral, and possible change of infiltration resulting from the heating of the soil. Analysis of the effects of these variables is beyond the scope of this study. Of importance is the fact that following the apparent increase in streamflow in 1983 and 1984, there was no increase in 1985 and 1986. Also, the flow in 1985 was greater than in 1984; in 1986, flow was less than that in 1984. Thus, even if the effect of the burn on annual discharge is nonlinear, it is likely that the quantity of annual discharge of the East Fork returned to preburn conditions about 3 years after the burn. One means of examining the possible nonlinear effects of the burn on streamflow is to separate annual discharge into periods of storm runoff.

\section{Storm Runoff}

Streamflow for the postburn period was separated into periods of storm runoff using methods developed by Hjalmarson (1975). Runoff resulting from a particular storm(s) was defined using hydrograph-separation techniques. The use of periods of storm runoff provides more samples and thereby can enhance the detection of changes in discharge, providing the variance of the sample does not increase greatly.

The separation of streamflow into periods of storm runoff provided 25 samples for the preburn period that were used to define the streamflow relation between the basins. The relation for storm runoff defined by Hjalmarson $(1975$, p. 72$)$ is

$$
\log Y=0.23+0.51(\log X)+0.13(\log X)^{2},
$$

where

$$
\begin{aligned}
& Y= \text { storm runoff, in acre-feet, East Fork } \\
& \text { basin, and } \\
& X= \text { storm runoff, in acre-feet, West Fork } \\
& \text { basin, and }
\end{aligned}
$$

For equation $2, R^{2}$ is 0.94 , and the standard error is $0.2249 \log$ units.

The method of hydrographic separation used by Hjalmarson (1975, Appendix C) was used with one modification. The average recession slope used to separate periods of runoff in the East Fork drainage basin during water years 1983 and 1984 was less steep than the average recession slope used to separate base flows during the preburn period. The flatter average recession slope for 1983-84 indicates that base flow increased in the immediate postburn period because less water stored in the ground was consumed by the vegetation in the East Fork basin. The average recession slope used for hydrographic separations in water years 1985-86 returned to that used for the preburn period. Twenty-four separate periods of runoff (fig. 5 and table 3) were computed for the postburn period using the hydrographic-separation technique.

Storm runoff for the East Fork basin was computed using equation 2 and the corresponding quantity of storm runoff for the West Fork basin (table 3). All the computed quantities of streamflow for the first 10 storm periods (first 2 water years of the postburn period) were less than the corresponding measured quantities of streamflow. The difference in streamflow was significant at the 68-percent probability level for 8 of the first 10 storm periods. Only storm periods 7 and 17 show an apparent increase of streamflow as a result of the burn at the 95 -percent prediction probability level (two standard deviations). There was significantly (95-percent probability level) less measured 


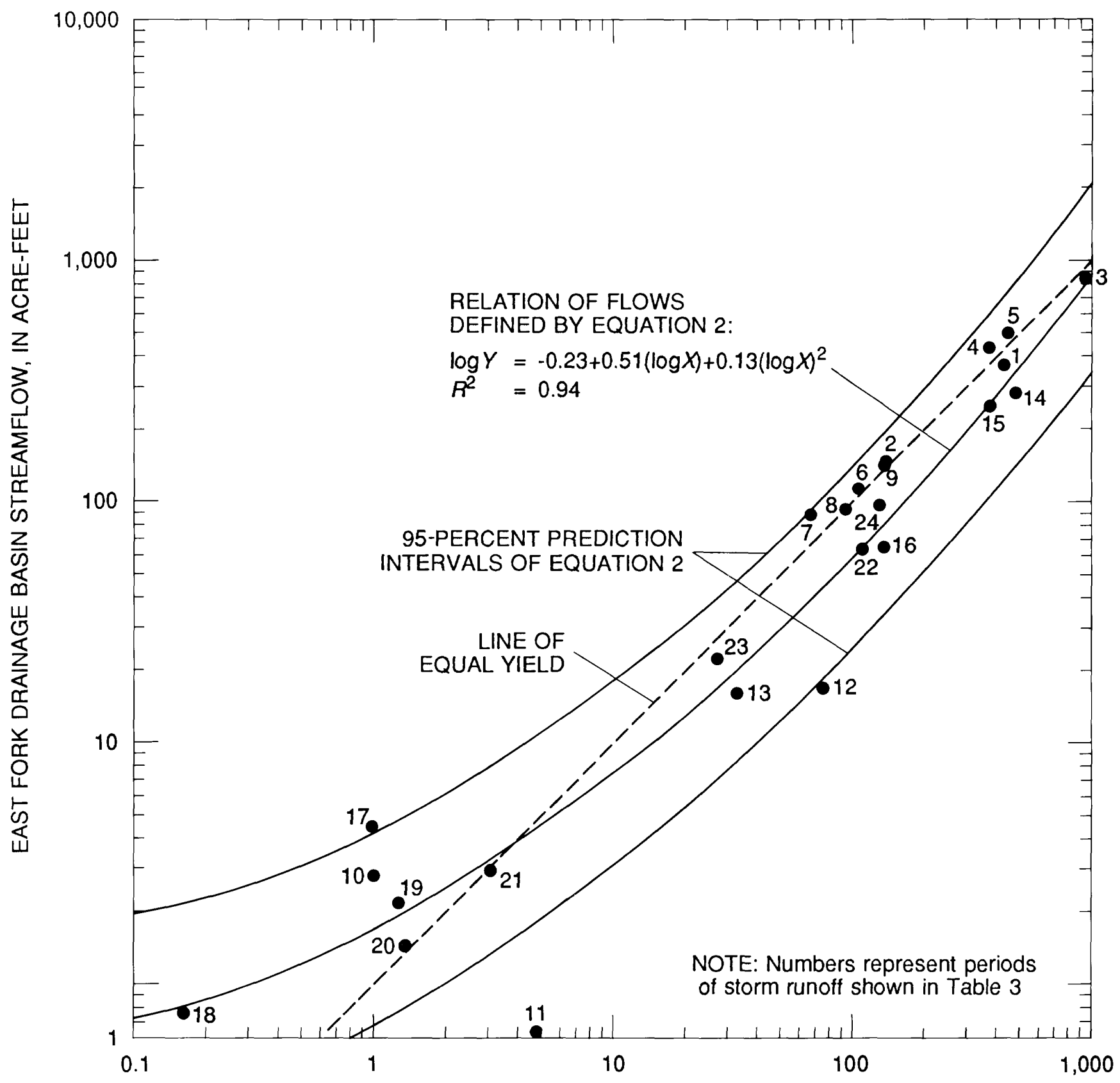

WEST FORK DRAINAGE BASIN STREAMFLOW, IN ACRE-FEET

Figure 5. Postburn storm runoff in East Fork and West Fork Sycamore Creek drainage basins, water years 1983-86. 
Table 3. Measured and computed streamflow for selected postburn periods of storm runoff, precipitation totals, and statistical-significance levels, East Fork and West Fork Sycamore Creek drainage basins, October 1982 through May 1986

\begin{tabular}{|c|c|c|c|c|c|c|c|c|c|}
\hline \multirow{3}{*}{$\begin{array}{l}\text { Period } \\
\text { of } \\
\text { storm } \\
\text { runoff }\end{array}$} & & & \multicolumn{2}{|c|}{ West Fork } & \multicolumn{5}{|c|}{ East Fork } \\
\hline & \multicolumn{2}{|c|}{ Date } & \multirow{2}{*}{$\begin{array}{l}\text { Precipi- } \\
\text { tation, in } \\
\text { inches }\end{array}$} & \multirow{2}{*}{$\begin{array}{l}\text { Measured } \\
\text { stream- } \\
\text { flow, } \\
\text { in } \\
\text { acre-feet }\end{array}$} & \multirow{2}{*}{$\begin{array}{l}\text { Precipi- } \\
\text { tation, in } \\
\text { inches }\end{array}$} & \multirow{2}{*}{$\begin{array}{l}\text { Measured } \\
\text { stream- } \\
\text { flow, } \\
\text { In } \\
\text { acre-feet }\end{array}$} & \multirow{2}{*}{$\begin{array}{l}\text { Computed } \\
\text { stream- } \\
\text { flow, } \\
\text { in } \\
\text { acre-feet }\end{array}$} & \multicolumn{2}{|c|}{$\begin{array}{l}\text { Difference between } \\
\text { measured and } \\
\text { computed streamflow }\end{array}$} \\
\hline & From & To & & & & & & Acre-feet & Percent \\
\hline 1 & $10-26-82$ & $12-21-82$ & 13.65 & 424 & 10.20 & 367 & 293 & 74.0 & 25 \\
\hline 2 & $12-22-82$ & $01-26-83$ & 3.00 & 138 & 2.52 & 146 & 82.5 & ${ }^{1} 63.5$ & ${ }^{177}$ \\
\hline 3 & $01-27-83$ & $03-02-83$ & 8.88 & 948 & 8.04 & 832 & 795 & 37 & 5 \\
\hline 4 & $03-03-83$ & $03-17-83$ & 4.20 & 375 & 3.36 & 426 & 254 & ${ }^{1} 172$ & ${ }^{1} 68$ \\
\hline 5 & $03-18-83$ & $04-17-83$ & 4.92 & 451 & 4.20 & 498 & 316 & ${ }^{1} 182$ & 158 \\
\hline 6 & $04-18-83$ & $09-25-83$ & 6.12 & 104 & 8.88 & 111 & 61.3 & ${ }^{1} 49.7$ & ${ }^{1} 81$ \\
\hline 7 & $09-26-83$ & $11-19-83$ & 8.16 & 67.5 & 6.72 & 88.6 & 39.6 & ${ }^{2} 49$ & ${ }^{2} 124$ \\
\hline 8 & $11-20-83$ & $12-24-83$ & 3.84 & 93.0 & 2.76 & 93.4 & 54.7 & ${ }^{1} 38.7$ & ${ }^{1} 30$ \\
\hline 9 & $12-25-83$ & $07-04-84$ & 5.40 & 136 & 5.28 & 142 & 81.3 & ${ }^{1} 60.7$ & ${ }^{1} 75$ \\
\hline 10 & $07-19-84$ & $08-25-84$ & 5.18 & .99 & 6.60 & 2.8 & 1.69 & ${ }^{1} 1.11$ & ${ }^{1} 66$ \\
\hline 11 & $08-26-84$ & $09-24-84$ & 2.04 & 4.78 & .24 & .63 & 4.33 & ${ }^{2}-3.70$ & $2-85$ \\
\hline 12 & $09-25-84$ & $11-22-84$ & 8.88 & 75.8 & 5.88 & 16.0 & 44.5 & ${ }^{2}-28.5$ & $2^{2}-64$ \\
\hline 13 & $11-23-84$ & $12-07-84$ & 3.84 & 32.6 & 3.36 & 16.2 & 19.9 & -3.7 & -19 \\
\hline 14 & $12-31-84$ & $01-25-85$ & 9.48 & 479 & 8.40 & 274 & 339 & -65 & -19 \\
\hline 15 & $01-26-85$ & $03-10-85$ & 5.04 & 381 & 4.80 & 246 & 258 & -12 & -5 \\
\hline 16 & $03-11-85$ & $07-06-85$ & 4.80 & 136 & 3.72 & 64.4 & 81.2 & -16.8 & -21 \\
\hline 17 & $07-07-85$ & $08-16-85$ & 3.60 & 1.01 & 3.48 & 4.48 & 1.71 & 22.77 & ${ }^{2} 162$ \\
\hline 18 & $08-17-85$ & $09-05-85$ & 2.40 & .16 & .36 & .77 & .81 & -.04 & -4 \\
\hline 19 & $09-06-85$ & $10-08-85$ & 2.40 & 1.25 & 2.88 & 2.20 & 1.91 & .29 & 15 \\
\hline 20 & $10-09-85$ & $11-11-85$ & 2.28 & 1.23 & 2.16 & 1.33 & 1.89 & ${ }^{1}-.56$ & ${ }^{1}-30$ \\
\hline 21 & $11-12-85$ & $11-24-85$ & 2.28 & 3.09 & 1.44 & 2.98 & 3.24 & -.26 & -8 \\
\hline 22 & $11-25-85$ & $01-29-86$ & 6.24 & 108 & 4.68 & 61.3 & 63.8 & -2.5 & -4 \\
\hline 23 & $01-30-86$ & $03-09-86$ & 3.60 & 27.4 & 3.72 & 22.7 & 17.1 & 15.6 & ${ }^{1} 33$ \\
\hline 24 & $03-10-86$ & $05-31-86$ & 5.40 & 130 & 4.68 & 96.7 & 77.5 & 19.2 & 25 \\
\hline
\end{tabular}

${ }^{1}$ Change significant at 68 -percent probability level.

${ }^{2}$ Change significant at 95 -percent probability level.

streamflow than computed streamflow for storm periods 11 and 12. Following the first 10 periods, there was less measured streamflow than computed streamflow for 10 of the 14 storm periods (periods 11-24).

Although precipitation differences between the basins are not statistically significant in the relation of storm runoff for the basins, some of the difference between the measured and computed streamflow for the East Fork basin may be related to precipitation differences. For example, for storm periods $1,11,12,18$, and 21 , more than the normal difference in precipitation occurred between the
West Fork and East Fork basins (table 3). Some of the precipitation that produced these periods of streamflow apparently missed the East Fork basin. Measured streamflow for West Fork may be larger than the measured streamflow for East Fork as a result of the precipitation differences. The result may be smaller-than-normal differences between measured streamflow and computed streamflow for East Fork for these five periods of storm runoff (table 3).

The burning of the vegetation may have less effect on runoff from the large storms. For example, for 3 of the first 10 storm periods with the largest 
quantity of runoff for the West Fork basin, the percentage of apparent increase of runoff for the East Fork basin was smaller than the increase for the other seven smaller periods. Only one of the three largest storm periods showed an increase of runoff at the 68-percent probability level; however, all of the seven smaller storm periods showed an increase at the 68-percent probability level.

Because 8 of the first 10 storm periods following the burn show an increase in runoff for the East Fork basin and subsequent storm periods show runoff amounts that appear to scatter about the preburn-streamflow relation (fig. 5), a short-lived increase of runoff is considered to have resulted from the burn. The scatter of the runoff from the postburn storm periods about the postburnstreamflow relation is large and tends to mask the detection of change. A visual examination of the plot of runoff for the postburn storm periods indicates that the variance is larger than the variance for the preburn relation and that the streamflow is not stationary.

Thus far, the apparent nonstationarity of the periods of storm runoff has not been evaluated using statistical methods. Because the postburn relation of the storm runoff between the basins appears to be different from the preburn relations and appears to be changing with time, a time variable was added to the regression analysis (Hirsch and others, 1982) as follows:

$$
\begin{aligned}
\log Y= & a+b(\log X)+c(\log X)^{2} \\
& +d[\log X(\text { TIME })],
\end{aligned}
$$

where

$$
\begin{aligned}
Y= & \text { storm runoff, in acre-feet, East Fork } \\
& \text { drainage basin; } \\
X= & \text { storm runoff, in acre-feet, West } \\
& \text { Fork drainage basin; } \\
T I M E= & \text { date of first day of the period of } \\
& \text { storm runoff in decimal time format } \\
& \text { minus } 1900 ; \text { and }
\end{aligned}
$$

A significant value of $d$ suggests that the relation is not stationary and has changed as a result of the burn. Two analyses were conducted using equation 3. For the first analysis, periods of storm runoff from the preburn period and the first 10 postburn runoff periods, which occurred between October 1, 1982, through August 1984, were used in the regression equation. The postburn periods of runoff for the analysis were selected because they had shown an apparent increase in storm runoff. The regression coefficient, $d$, was significant at the 98-percent probability level, indicating that the burn caused an increase in streamflow for the East Fork basin.

A second analysis was done using the preburn record and the postburn periods of storm runoff that were not used in the first analysis. The postburn periods of storm runoff, which occurred from September 1, 1984, through May 31, 1986, had not shown an apparent increase in streamflow. The value of the regression coefficient, $d$, computed in the second analysis was not significant. The results indicate that the postburn storm runoff during the fourth and fifth years after the burn was statistically equivalent to storm runoff in the preburn period. Thus, there was an increase in streamflow for the second and third water years after the burn, as indicated by the results of the first analysis, but in subsequent years, streamflow in the East Fork basin returned to preburn levels.

\section{Instantaneous Peak Discharge}

Although the burn may not have affected the streamflow in wet years and larger occurrences of runoff, a more detailed analysis was made to determine if the burn had an effect on the magnitude of instantaneous peak flow. A Wilcoxon signed rank test (Inman and Conover, 1983, p. 256) was used on selected instantaneous peak discharges of more than $10 \mathrm{ft}^{3} / \mathrm{s}$ for both basins (table 4 ). The base instantaneous peak discharge of $10 \mathrm{ft}^{3} / \mathrm{s}$ was selected using criteria by Novak (1985, p. 92). The null hypothesis used in this test is that the mean of the instantaneous peak discharges in the East Fork basin is equal to the mean of the instantaneous peak discharges in the West Fork basin. The test was 
Table 4. Selected peak discharges and peak yields at the streamflow-gaging stations, East Fork and West Fork Sycamore Creek drainage basins, water years 1965-86

\begin{tabular}{|c|c|c|c|c|}
\hline \multirow[b]{2}{*}{ Date } & \multicolumn{2}{|c|}{$\begin{array}{c}\text { East Fork } \\
\text { Sycamore Creek }\end{array}$} & \multicolumn{2}{|c|}{$\begin{array}{c}\text { West Fork } \\
\text { Sycamore Creek }\end{array}$} \\
\hline & $\begin{array}{l}\text { Peak } \\
\text { dis- } \\
\text { charge, } \\
\text { in } \\
\text { cubic } \\
\text { feet per } \\
\text { second }\end{array}$ & $\begin{array}{c}\text { Peak } \\
\text { yield, in } \\
\text { cubic } \\
\text { feet per } \\
\text { second } \\
\text { per } \\
\text { square } \\
\text { mile }\end{array}$ & $\begin{array}{l}\text { Peak } \\
\text { dis- } \\
\text { charge, } \\
\text { in } \\
\text { cubic } \\
\text { feet per } \\
\text { second }\end{array}$ & $\begin{array}{c}\text { Peak } \\
\text { yield, in } \\
\text { cubic } \\
\text { feet per } \\
\text { second } \\
\text { per } \\
\text { square } \\
\text { mile }\end{array}$ \\
\hline December 10,1965 & 100 & 22.1 & 68 & 14.7 \\
\hline December 22, 1965 & 330 & 73.0 & 430 & 92.3 \\
\hline December 30, 1965 & 180 & 39.8 & 141 & 30.4 \\
\hline December 19, 1967 & 244 & 54.0 & 152 & 32.8 \\
\hline January 28,1968 & 104 & 23.0 & 69 & 14.9 \\
\hline February 14, 1968 & 20 & 4.42 & 22 & 4.74 \\
\hline March 9, 1968 & 20 & 4.42 & 10 & 2.16 \\
\hline September 5, 1970 & 1,940 & 429 & 3,480 & 750 \\
\hline October 7, 1972 & 125 & 27.7 & 185 & 39.9 \\
\hline October 19,1972 & 52 & 11.5 & 51 & 11.0 \\
\hline November 17,1972 & 25 & 5.53 & 22 & 4.74 \\
\hline December 28, 1972 & 84 & 18.6 & 84 & 18.1 \\
\hline February 21,1973 & 42 & 9.29 & 33 & 7.11 \\
\hline March 17, 1973 & 36 & 7.96 & 31 & 6.68 \\
\hline March 31, 1973 & 38 & 8.41 & 34 & 7.33 \\
\hline November 30,1982 & 157 & 34.7 & 178 & 38.4 \\
\hline December 10,1982 & 39 & 8.63 & 36 & 7.76 \\
\hline December 23, 1982 & 80 & 17.7 & 35 & 7.54 \\
\hline January 29,1983 & 84 & 18.6 & 115 & 29.8 \\
\hline February 8,1983 & 146 & 32.3 & 155 & 33.4 \\
\hline March 4, 1983 & 83 & 18.4 & 48 & 10.3 \\
\hline $\operatorname{March} 24,1983$ & 67 & 14.8 & 47 & 10.1 \\
\hline October 1,1983 & 45 & 9.96 & 21 & 4.52 \\
\hline December 4, 1983 & 26 & 5.75 & 12 & 2.59 \\
\hline December 27, 1983 & 64 & 14.2 & 21 & 4.53 \\
\hline October 3, 1984 & 42 & 9.29 & 10 & 2.16 \\
\hline December 27, 1984 & 78 & 17.3 & 97 & 20.9 \\
\hline November 30,1985 & 12 & 2.65 & 15 & 3.23 \\
\hline
\end{tabular}

performed separately on the preburn data and the postburn data. The null hypothesis of the Wilcoxon test could not be rejected for both the preburn and postburn periods. The burn, therefore, did not change the magnitude of flood-peak discharge for the East Fork basin.

Similar results were obtained by analyzing instantaneous peak-discharge data using multiple- regression analysis and a time variable. Peak flows measured on the East Fork basin were regressed against peak flows on the West Fork basin. The peak flow on the West Fork basin was multiplied by the date of the peak minus 1900 to form the following equation:

$$
\begin{gathered}
\log Y=0.540+0.804(\log X) \\
-0.00072[\log X(\text { TIME })],
\end{gathered}
$$

where

$$
\begin{aligned}
Y= & \text { instantaneous peak discharge, in cubic } \\
& \text { feet per second, East Fork drainage } \\
& \text { basin; } \\
X= & \text { instantaneous peak discharge, in cubic } \\
& \text { feet per second, West Fork drainage } \\
& \text { basin; and } \\
T I M E= & \text { date of peak in decimal time format } \\
& \text { minus } 1900 .
\end{aligned}
$$

The coefficient for the time factor was not significant; therefore, peak flows for the postburn period were not significantly different from those for the preburn period. Peak-flow data for 1985-86 were excluded from the data set, and the regression analysis was redone with similar results.

\section{Duration of Streamflow}

Most of the streamflow at the East Fork and West Fork Sycamore Creek streamflow-gaging stations during the preburn period of the study occurred from November to April of each year. Although 54 percent of the total annual precipitation on the two drainage basins was recorded during this 6-month period (fig. 3), 87 percent of the total streamflow was recorded during this period. The remaining 46 percent of annual precipitation that occurred from May to October produced only 13 percent of the annual streamflow, indicating that most of the rainfall during the summer was lost to evapotranspiration or replenished soil moisture.

Distributions of daily discharge for each basin were combined into flow-duration curves for the preburn and 1983-84 periods to compare the effects 
of the burn on streamflow characteristics. Daily discharge data for water years 1985-86 were not used in the computation of flow-duration curves for the postburn period because the results of the regression analysis discussed earlier generally showed no increase in streamflow for this period on East Fork.

Flow-duration curves for the preburn period for the East Fork and West Fork basins are similar in shape and slope (fig. 6). These curves show that flows in the West Fork basin generally are larger than those in the East Fork basin when daily flow in both streams is between about 0.02 and $20 \mathrm{ft}^{3} / \mathrm{s}$. The lower ends of the curves show that East Fork has a higher percentage of days with flows of less than $0.01 \mathrm{ft}^{3} / \mathrm{s}$.

The low-flow end of the postburn curve for the East Fork basin changed markedly, and flow was perennial for the 2-year period. The displacement of the postburn relations is because daily streamflow quantities were greater during the postburn period than during the preburn period for both basins. Above about $0.6 \mathrm{ft}^{3} / \mathrm{s}$, the postburn curves are nearly the same, indicating a change in flow of a quantity equal to the displacement between the preburn curves. Between about 0.09 and $0.6 \mathrm{ft}^{3} / \mathrm{s}$, a shift occurred in the relation between the basins, and streamflow in the East Fork basin within that range of discharge was greater than streamflow in the West Fork basin.

The previous observations are made with the knowledge that the comparison of flow-duration curves for short periods of flow has pitfalls. The natural variation of daily streamflow can be large and can mask the true effects of the burn on streamflow. Also, when only 2 years of daily flow are used to define the postburn curve, the effect of discharge-measurement and computation errors can affect the shape of parts of the curve. The above observations, however, tend to support the previous quantitative analyses.

\section{Springs}

During the postburn period, Thicket Spring in the north-central part of the West Fork drainage basin (pl. 1) and Horse Camp Seep in the northern part of the East Fork drainage basin were measured once a year. Monthly measurements were made at Oneda Mine Spring where vegetation in part of the potential source area was burned. During postburn, measuring conditions at the Oneda Mine Spring were poor. Statistical summaries of measured discharge for the three springs during both periods of the study were computed for each site (table 5). No discernible change in the spring flow at Horse Camp Seep and Oneda Mine Spring could be attributed to the change in vegetative cover as a result of the burn of October 1981. Flow from Horse Camp Seep would not be expected to change as the seep is upgradient from the area reached by the fire. Mean flow rates at Oneda Mine Spring were the same for both periods of the study.

\section{Effects of Burning on Sediment Transport and Deposition}

Basins with recently burned vegetation have experienced large amounts of soil movement and sedimentation. Measurement of total sediment yield is difficult because of the problem of measuring the amount of material moving along the channel beds. For this study, three methods were used to estimate the change in erosion rates and the sedimentation of

Table 5. Range in measured discharge at Thicket and Oneda Mine Springs and Horse Camp Seep

[Dashes indicate no data]

\begin{tabular}{l|c|c|c|c|c|c}
\hline \multirow{2}{*}{ Spring } & \multicolumn{4}{c|}{ Measured discharge, in cubic feet per second } \\
\cline { 2 - 7 } & \multicolumn{3}{c|}{ Preburn period } & \multicolumn{4}{c}{ Postburn period } \\
\cline { 2 - 7 } & Minimum & Maximum & Mean & Minimum & Maximum & Mean \\
\hline Thicket Spring ............... & -- & - & 0.001 & 0 & 0.0006 & 0.0002 \\
Oneda Mine Spring ......... & 0 & 0.007 & .002 & .0005 & .004 & .002 \\
Horse Camp Seep........... & .00003 & .007 & .001 & .00006 & .001 & .002 \\
\hline
\end{tabular}




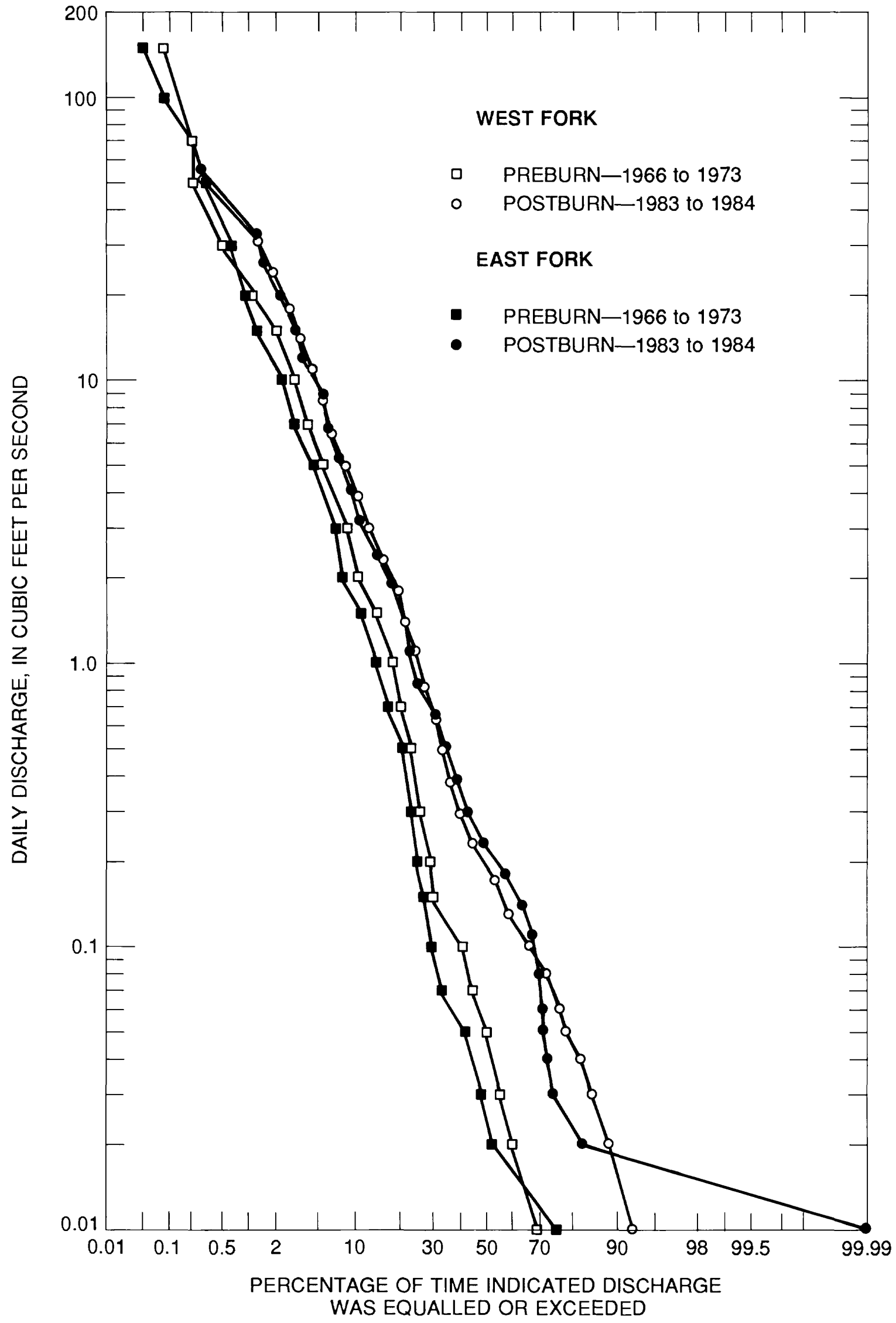

Figure 6. Flow-duration curves for East Fork and West Fork Sycamore Creek, water years 1966-73 and 1983-84. 
the East Fork basin as a result of the burn. First, the annual suspended-sediment yield was estimated before and after the burn for both basins. Second, an index of soil erosion was obtained at hillside transects in the basins. Third, a measure of channel aggradation and degradation in and downstream from the basins along the main-stream channels was obtained at channel cross sections. These three methods and visual observations of the physical characteristics of the hillside and channel material of the basins were used to estimate the change, if any, in sediment yield for the East Fork basin.

In the first method, data collected at the suspended-sediment sampling sites at the streamflow-gaging stations were used to estimate the amount of suspended sediment that was transported from the study area. The suspendedsediment yield for both basins was calculated from measurements of suspended-sediment concentrations and stream discharge using standard techniques and methods (Porterfield, 1972).

The second method was designed to measure changes, if any, in the topsoil layer in the East Fork basin. Hillside transects were established in the north half of each drainage basin away from the major stream channels.

The third method used changes in channel geometry, as defined by periodic surveys, of channel cross sections as an index of sediment movement along the main channels of the drainage basins. Stable channel cross-sectional geometry indicates no erosion and no change in runoff from upstream basins.

\section{Suspended-Sediment Yields}

Annual suspended-sediment yields for the East Fork and West Fork drainage basins were estimated to be 200 tons $/ \mathrm{mi}^{2}$ for the preburn period, water years 1966-73. The estimated yields were computed from data that were insufficient to fully define the relation between sediment concentration and streamflow (Hjalmarson, 1975, p. 32). The low-flow parts of the graphs were determined from suspended-sediment samples and streamflow measurements. Suspended-sediment concentrations for high flows were estimated primarily from a few fixed-stage samples and a few manually collected samples. Streamflow quantities for high flows were based on the stage-discharge relations at each streamflow-gaging station that were adequately defined by slope-area measurements. During the preburn period, some mining activity was occurring in both basins that probably had some effect on the amount of sediment being transported in the channels. Estimated suspended-sediment yield for the preburn period includes the effect of traffic from mining activities on two small roads located in the stream channels upstream from the sedimentsampling sites. The effect of this traffic on the sediment yield of the basins during the preburn period is unknown but probably increased the sediment yield of the basins.

Annual suspended-sediment yields for both basins during the postburn period were computed from daily suspended-sediment loads calculated for each station using suspended-sediment concentrations and streamflow hydrographs (table 6, fig. 7). The hydrographs were based on streamflow records and samples of suspended-sediment concentrations. Suspended-sediment loads were not calculated when mean streamflow was less than 2.0 $\mathrm{ft}^{3} / \mathrm{s}$ because (1) orifice intakes were set to sample streamflows $2.0 \mathrm{ft}^{3} / \mathrm{s}$ and greater to prevent clogging with bottom material and (2) suspended-sediment concentrations for flows of less than $2.0 \mathrm{ft}^{3} / \mathrm{s}$ generally were less than $5 \mathrm{mg} / \mathrm{L}$ and were not a significant component of annual suspended-sediment yield.

The computed suspended-sediment yield for the East Fork basin was much greater than the suspended-sediment yield for the West Fork basin for the postburn years of 1983 and 1984. The suspended-sediment yield was 24 and 88 times more for the East Fork basin than for the West Fork basin for 1983 and 1984, respectively. For 1985 and part of water year 1986, there was only 2.8 and 2.6 times more yield, respectively, for the East Fork basin. Because of the poorly defined computed suspended-sediment yield for the preburn period, it is unknown if the ratios of suspended-sediment yield for 1985 and 1986 represent a return to preburn conditions. On the basis of changes in 


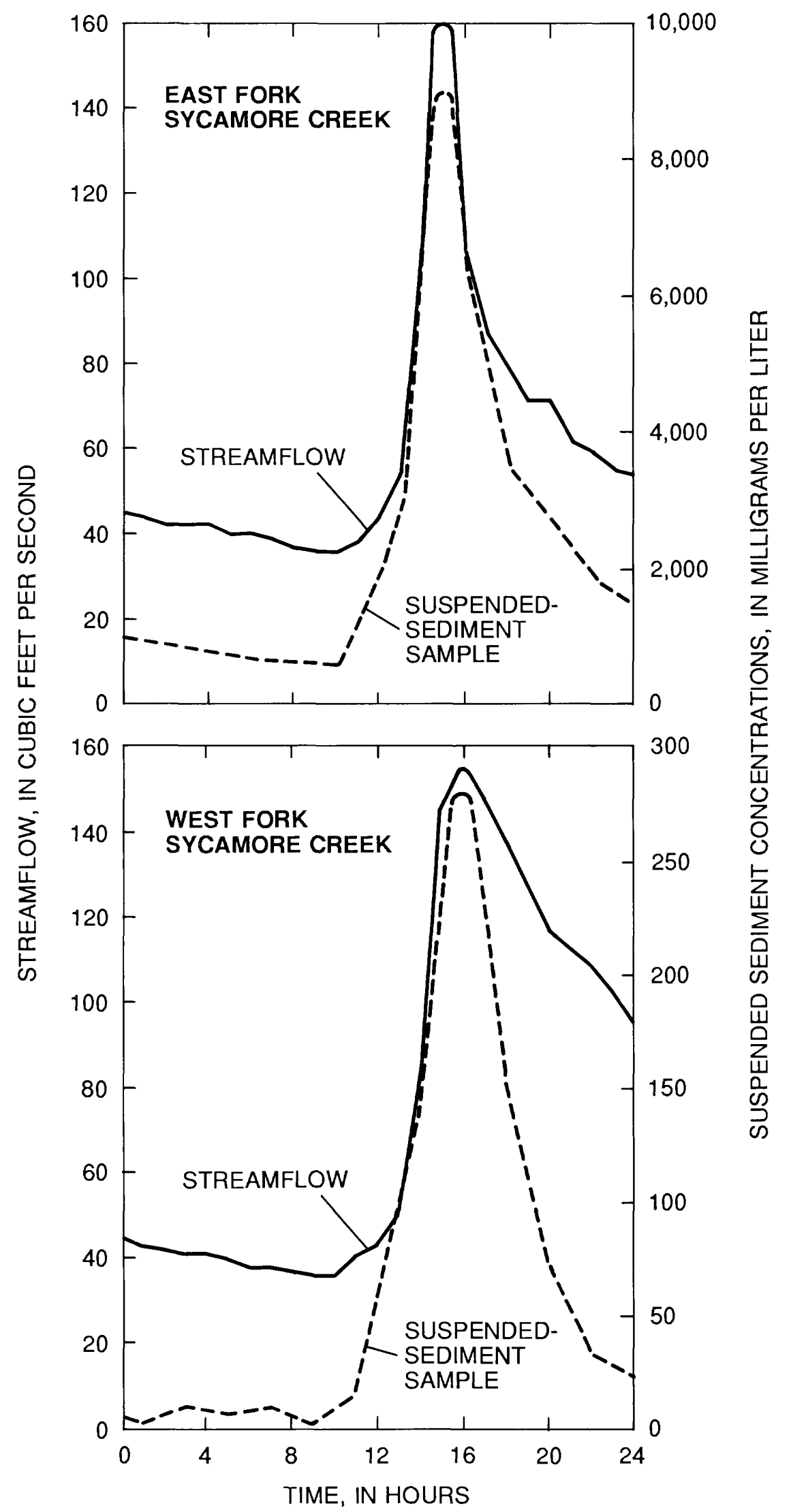

Flgure 7. Streamflow and suspended-sediment concentrations, East Fork and West Fork Sycamore Creek, February 8, 1983. 
Table 6. Annual suspended-sediment loads and yields, East Fork and West Fork Sycamore Creek drainage basins, water years 1983-86

\begin{tabular}{|c|c|c|c|c|c|}
\hline \multirow[b]{3}{*}{$\begin{array}{c}\text { Water } \\
\text { year }\end{array}$} & \multicolumn{4}{|c|}{ Suspended sediment } & \multirow[b]{3}{*}{$\begin{array}{c}\text { Ratio of } \\
\text { East Fork to } \\
\text { West Fork } \\
\text { suspended- } \\
\text { sediment } \\
\text { yield }\end{array}$} \\
\hline & \multicolumn{2}{|c|}{ East Fork } & \multicolumn{2}{|c|}{ West Fork } & \\
\hline & $\begin{array}{c}\text { Load, in } \\
\text { tons }\end{array}$ & $\begin{array}{l}\text { Yield, in } \\
\text { tons } \\
\text { per } \\
\text { square } \\
\text { mile }\end{array}$ & $\begin{array}{l}\text { Load, in } \\
\text { tons }\end{array}$ & $\begin{array}{c}\text { Yield, } \\
\text { in tons } \\
\text { per } \\
\text { square } \\
\text { mile }\end{array}$ & \\
\hline 1983 & 2,470 & 546 & 105 & 22.6 & $24: 1$ \\
\hline 1984 & 303 & 67.1 & 3.51 & .76 & $88: 1$ \\
\hline 1985 & 173 & 38.3 & 61.6 & 13.3 & $2.8: 1$ \\
\hline $1986^{1}$ & 11.7 & 2.58 & 4.62 & 1.00 & $2.6: 1$ \\
\hline
\end{tabular}

${ }^{1}$ Partial year, October 1 through May 31, 1986.

suspended-sediment yield after a burn on the Three Bar experimental watershed (fig. 8), the ratios for 1985-86 (table 6) probably are greater than the prebum ratios because it appears to take at least 6 years to return to near-preburn conditions. If the ratios for 1985-86 represent preburn levels, then the apparent increase of suspended-sediment yield as a result of the burn is about 9 times for 1983 and 33 times for 1984 . If the preburn ratio of suspended-sediment yield for the basins was $1: 1$ as indicated by the preburn estimates, then the apparent increase of suspended-sediment yield for the East Fork basin is 24 times for 1983 and 88 times for 1984.

Studies by other researchers indicate that the decreasing sediment yield from the East Fork drainage basin for water years 1983-86 is typical of chaparral-covered drainage basins for the second to fourth years after a fire. Suspended-sediment yield on the Three Bar experimental watershed in central Arizona during the first year after a fire was typified by a large increase in sediment yield followed by a rapid decrease in following years (Hibbert and others, 1975, p. 445-468). The suspended-sediment yields for 1983-85 for East Fork of 14.2, 1.7, and $1.0 \mathrm{ft}^{3} /$ acre were calculated using an average unit weight (Potter, 1967, p. 1133) of $120 \mathrm{lbs} / \mathrm{ft}^{3}$ determined from sieve analyses of suspendedsediment samples. The suspended-sediment yields for the East Fork basin and for the Three Bar experimental watershed decrease greatly for the second through fourth years after the fire (fig. 8).
Suspended-sediment data were not collected immediately before the controlled burn nor for 1 year after the burn on the East Fork basin; therefore, suspended-sediment yields could not be compared with data from the Three Bar experimental watershed for this period.

An equation was developed using generalized least-squares regression methods to determine the relation of suspended-sediment concentration to streamflow from the East Fork drainage basin during rising streamflow associated with winter storms during the postburn period. The computed regression equation for streamflows of 2 to $100 \mathrm{ft}^{3} / \mathrm{s}$ is

$$
\log E F S c=1.45+0.757(\log E F Q),
$$

where

$$
\begin{aligned}
E F S c= & \text { East Fork suspended-sediment con- } \\
& \text { centration, in milligrams per liter, and } \\
E F Q= & \text { East Fork discharge, in cubic feet per } \\
& \text { second. }
\end{aligned}
$$

The standard error for equation 5 is 0.4398 log units with a $\mathrm{R}^{2}$ of 0.375 . The data set used to compute equation 5 was restricted to rising stream stages during winter storms and did not include suspended-sediment samples collected during falling stages of a period of runoff. Equation 5 was analyzed to determine if a change occurred in sediment-transport characteristics as a result of regrowth of the chaparral by using an analysis-of-covariance test with a dummy variable (Draper and Smith, 1981, p. 241-257).

The analysis-of-covariance test discussed earlier, which was based on streamflow data collected during water years 1983-84, showed an increase in streamflow over predicted levels on the East Fork drainage basin. The test made on 1985-86 data showed a return to preburn flow conditions. The change in flow conditions led to the hypothesis that the vegetation had regrown enough to return streamflow patterns to prebum conditions during and after water year 1985. A dummy variable with a value of 0 or 1 was then 


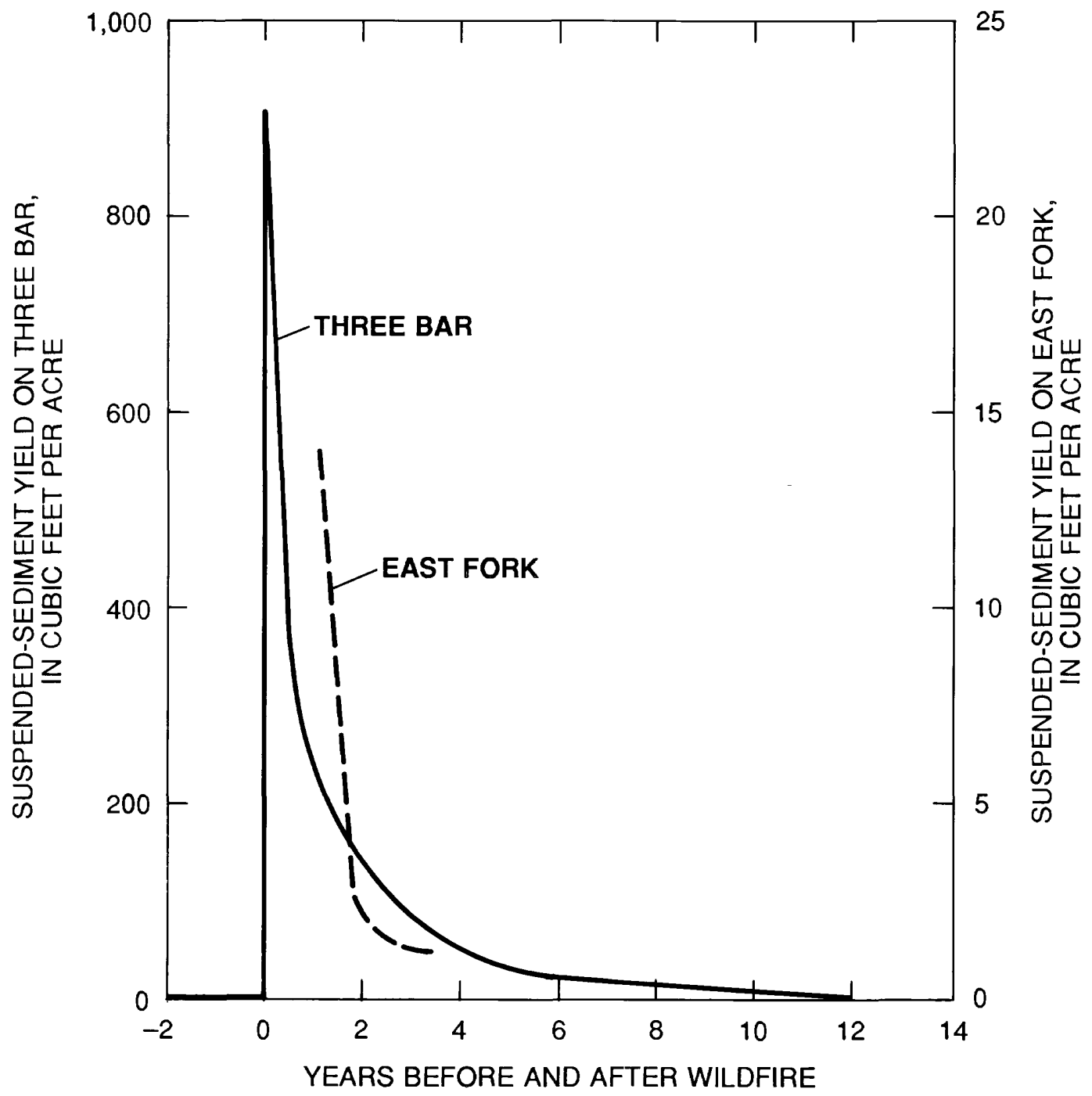

Figure 8. Mean suspended-sediment yield on the Three Bar experimental watershed before and after controlled burning in 1959 and mean suspended-sediment yield on East Fork Sycamore Creek after wildfire in 1981 (Hibbert and others, 1975 , p. $445-468)$.

added to the East Fork regression (eq. 5). Zero values were assigned to the dummy variable for flows prior to water year 1985. A value of 1 was assigned to observations for water years 1985-86. The null hypothesis states that the regrowth of the vegetation had no effect on the relation between suspended-sediment concentrations and streamflow. The analysis-of-covariance test indicated that the null hypothesis was accepted at a confidence level of 95 percent and no significant change occurred in the intercept or slope of the East Fork regression line (eq. 5). The results of this test indicate that the increase in sediment yield during the first 2 years of the postburn period probably was caused by the increase in streamflow resulting from the burn rather than from a change in sediment concentrations per unit discharge of the East Fork drainage basin. 


\section{Hillside Transects}

The three hillside transects used for the preburn period were surveyed in the fall of 1983 for the first time since 1973 (p1. 1). On-site inspection of transect 1 on the West Fork drainage basin showed that the ground surface along the line was not affected by the controlled burn. Transect 2 in the East Fork basin was completely covered by the burn. The lower two-thirds of transect 3 was partly burned; the upper one-third showed no evidence of being burned. The main objective of reactivating data-collection activities on the three transects was to determine if colluvium was moving off the drainage basins as a result of the burn rather than in response to individual storms with high-intensity rainfall. Transect 1 is considered the control, and transect 2 is the affected (experimental) line. Data for transect 3 were not used in this analysis because the transect was only partly burned.
A one-way analysis-of-variance test (Inman and Conover, 1983, p. 408) was performed on the data collected on transects 1 and 2 for water years 1982-85 (table 7). A null hypothesis of no difference in the change in soil-elevation level between transects 1 and 2 and within each transect was tested at a 95-percent confidence level for the October 1982 measurement. From the analysisof-variance test, the null hypothesis could not be rejected for transects 1 and 2. Transect data collected between 1974 and 1982 also were tested with similar results, indicating that the hillsides where the transects are located are fairly stable and that large amounts of material did not move as a result of the burn. Visual inspections of both basins did not indicate an increase in frequency and degree of rills or widespread sloughing of colluvium in the burned area.

Table 7. Aggradation and degradation of colluvium, East Fork and West Fork Sycamore Creek drainage basins, water years $1983-85$

[See plate 1 for location of transects. Plus sign (+) denotes aggradation; minus sign (-) denotes degradation; dashes (-) indicate that site was not measured]

\begin{tabular}{|c|c|c|c|c|c|c|c|c|c|}
\hline \multirow{3}{*}{$\begin{array}{l}\text { Distance from } \\
\text { uppermost } \\
\text { measured } \\
\text { point, in feet }\end{array}$} & \multicolumn{9}{|c|}{ Change in altitude of the ground surface, in feet } \\
\hline & \multicolumn{3}{|c|}{1983} & \multicolumn{3}{|c|}{1984} & \multicolumn{3}{|c|}{1985} \\
\hline & $\begin{array}{l}\text { Tran- } \\
\text { sect } 1\end{array}$ & $\begin{array}{l}\text { Tran- } \\
\text { sect } 2\end{array}$ & $\begin{array}{l}\text { Tran- } \\
\text { sect } 3\end{array}$ & $\begin{array}{l}\text { Tran- } \\
\text { sect } 1\end{array}$ & $\begin{array}{l}\text { Tran- } \\
\text { sect } 2\end{array}$ & $\begin{array}{l}\text { Tran- } \\
\text { sect } 3\end{array}$ & $\begin{array}{l}\text { Tran- } \\
\text { sect } 1\end{array}$ & $\begin{array}{l}\text { Tran- } \\
\text { sect } 2\end{array}$ & $\begin{array}{l}\text { Tran- } \\
\text { sect } 3\end{array}$ \\
\hline 0 & +0.02 & +0.05 & +0.08 & +0.02 & +0.05 & +0.20 & -0.02 & +0.02 & -0.04 \\
\hline 15 & +.04 & -.05 & -.05 & +.01 & -.02 & +.15 & -.07 & +.02 & +.01 \\
\hline 30 & 0 & -.02 & -.06 & +.04 & -.02 & 0 & -.01 & -.02 & 0 \\
\hline 45 & -.01 & -.17 & +.04 & +.03 & 0 & +.02 & 0 & 0 & 0 \\
\hline 60 & 0 & +.01 & +.01 & +.01 & -.05 & -.01 & +.06 & -.03 & -.02 \\
\hline 75 & -.03 & +.04 & 0 & +.06 & +.05 & +.08 & -.01 & 0 & -.06 \\
\hline 90 & -.02 & -.02 & -.01 & +.05 & +.02 & +.09 & +.04 & +.01 & -.05 \\
\hline 105 & -.07 & -.12 & -.05 & -.03 & +.02 & +.03 & +.01 & -.02 & 0 \\
\hline 120 & -.03 & -.04 & 0 & +.03 & +.03 & -.03 & -.01 & +.02 & +.01 \\
\hline 135 & -.02 & -.01 & +.04 & -.01 & +.10 & -.04 & +.16 & -.02 & -.02 \\
\hline 150 & -.07 & 0 & 0 & +.03 & +.01 & +.04 & -.01 & +.01 & -.03 \\
\hline 165 & -.03 & -.03 & -.10 & +.04 & +.04 & +.06 & -.02 & -.05 & +.02 \\
\hline 180 & +.04 & -.17 & -.03 & -.05 & +.02 & +.07 & +.09 & -.04 & -.01 \\
\hline 195 & -.06 & +.02 & 0 & -.05 & +.01 & +.10 & +.01 & +.03 & -.02 \\
\hline 210 & -.04 & -.02 & +.03 & -.02 & +.06 & +.08 & +.01 & -.01 & 0 \\
\hline 225 & -.06 & -.03 & +.06 & 0 & +.05 & +.05 & +.02 & 0 & -.03 \\
\hline 240 & +.01 & +.04 & 0 & +.09 & -.03 & +.02 & -.01 & 0 & -.03 \\
\hline 255 & +.01 & -.07 & -.11 & +.04 & +.02 & +.02 & -.01 & -.03 & 0 \\
\hline 270 & 0 & -.. & 0 & +.05 & -.. & +.06 & +.02 & -.. & -.01 \\
\hline 285 & 0 & +.03 & +.01 & +.03 & +.02 & 0 & 0 & +.01 & -.02 \\
\hline
\end{tabular}




\section{Channel Cross Sections}

The average annual net change of cross-section area at the channel-monitoring sites (pl. 1) was nearly the same for the preburn and postburn periods (table 8). The average annual net change for the 19 sites used for the preburn period was $2.0 \mathrm{ft}^{2}$ of scour, and the average annual net change for the 12 sites used for the postburn period was $1.4 \mathrm{ft}^{2}$ of scour. The computed average amounts of channel scour and the computed difference of scour for the two periods are considered to be insignificant. On the basis of this small sample, no large net aggradation or degradation of the channels is considered to have occurred during the study.

Of particular interest are the measured changes in area at cross sections 7-15 along the East Fork basin channel (pl.1). The computed average annual net change for these sections was only $0.7 \mathrm{ft}^{2}$ and $0.3 \mathrm{ft}^{2}$ of fill, respectively, for the preburn and postburn periods. These small amounts of fill are considered insignificant. The burn apparently did not result in large changes of channel cross-section area along the main channel of East Fork Sycamore Creek.

The geometry of the surveyed cross sections was considered stable and did not reflect an increase of hillside erosion in the East Fork basin or an increase of sediment-transport rates from the East Fork basin as a result of the burn. A large increase of hillside erosion in the East Fork basin would have been expected to have changed the geometry of cross section 15 , which is closest to the burned area. The net change from 1982 to 1985 , however, was only $3 \mathrm{ft}^{2}$ of fill, which is considered insignificant.

Table 8. Changes in cross-sectional area at channel-change monitoring sites, East Fork and West Fork Sycamore Creek drainage basins and adjacent areas for indicated water years

[Dashes indicate that site was not measured]

\begin{tabular}{|c|c|c|c|c|c|c|c|c|c|c|c|c|}
\hline \multirow{2}{*}{$\begin{array}{l}\text { Cross } \\
\text { sec- } \\
\text { tion } \\
\text { (pl. 1) }\end{array}$} & \multicolumn{6}{|c|}{$\begin{array}{c}\text { Cross-sectional area, in square feet, for indicated } \\
\text { water year }\end{array}$} & \multirow{2}{*}{$\begin{array}{c}\text { Preburn } \\
\text { average } \\
\text { annual } \\
\text { net } \\
\text { change in } \\
\text { area }\end{array}$} & \multicolumn{4}{|c|}{$\begin{array}{l}\text { Cross-sectional area, in square feet, } \\
\text { for indicated water year }\end{array}$} & \multirow{2}{*}{$\begin{array}{l}\text { Postburn } \\
\text { average } \\
\text { annual net } \\
\text { change in } \\
\text { area }\end{array}$} \\
\hline & 1969 & 1970 & 1971 & 1972 & 1973 & 1974 & & 1982 & 1983 & 1984 & 1985 & \\
\hline 1 & $-\cdots$ & -.-- & ---- & 920 & 1,015 & 1,016 & 48 & $-\cdots$ & $\ldots$ & $\overline{-\cdots--}$ & ---- & ---- \\
\hline 2 & ----- & ---- & ---- & 972 & 1,004 & 947 & -12 & $\ldots$ & -.-. & -..-- & $-\cdots$ & $\cdots$ \\
\hline 3 & ---- & ---- & ----- & 674 & 685 & 691 & 8 & --- & -..- & --.-- & ---- & ---- \\
\hline 4 & 1,244 & ----- & 1,143 & 1,156 & 1,161 & 1,183 & -12 & 1,163 & 1,218 & 1,223 & 1,198 & 12 \\
\hline 5 & ---- & $-\cdots--$ & ----- & 470 & 464 & (1) & -6 & ---- & -..- & -... & $\cdots$ & $-\cdots$ \\
\hline 6 & 222 & -...- & 254 & 245 & 239 & 250 & +6 & 265 & 280 & 268 & 269 & 1 \\
\hline 7 & ----- & ---- & 129 & 132 & 146 & 146 & 6 & $\left({ }^{1}\right)$ & $-\cdots$ & $-\cdots-$ & $\cdots$ & -..... \\
\hline 8 & ---- & ---. & --.- & 1,550 & 1,550 & 1,511 & -20 & (2) & --- & $-\cdots$ & $-\cdots$ & $\ldots$ \\
\hline 9 & ---- & ----- & ---- & 433 & 438 & 450 & 8 & 480 & 478 & 483 & 480 & 0 \\
\hline 10 & 249 & --.-- & 254 & 252 & 246 & 251 & 0 & 277 & 277 & 276 & 275 & -1 \\
\hline 11 & --.-- & ---- & --..- & 122 & 118 & 122 & 0 & 112 & 113 & 118 & 116 & 1 \\
\hline 12 & 185 & ----- & 170 & 170 & 159 & 160 & -5 & 176 & 176 & 172 & 176 & 0 \\
\hline 13 & 265 & ---- & --.-- & 263 & 265 & 261 & -1 & --- & $\cdots$ & --.-- & 301 & $-\cdots-$ \\
\hline 14 & --.-- & ---- & $\cdots$ & 368 & 354 & 355 & -6 & 347 & 348 & 350 & 357 & 3 \\
\hline 15 & --.-- & --.-- & ---- & 351 & 580 & 576 & 12 & 596 & 596 & 605 & 593 & -3 \\
\hline 16 & 166 & ---- & 186 & 186 & 179 & 180 & 3 & 192 & 198 & 197 & 191 & 0 \\
\hline 17 & ---- & ---- & ---- & 503 & 490 & 473 & -15 & 514 & 520 & 515 & 519 & 2 \\
\hline 18 & -..-- & -...- & -..- & 821 & 864 & 881 & 30 & 843 & 840 & 828 & 837 & -2 \\
\hline 19 & $-\ldots .$. & 367 & -..-- & -...- & 367 & 368 & 0 & 356 & 363 & 361 & 363 & 2 \\
\hline Average & & & & & & & 2.0 & & & & & 1.4 \\
\hline
\end{tabular}

${ }^{\top}$ Bulldozer work in cross section, site discontinued.

${ }^{2}$ Reference marks lost, site discontinued. 


\section{SUMMARY AND CONCLUSIONS}

A study to determine effects of a controlled burn of chaparral vegetation was made on the East and West Forks of Sycamore Creek basin, which is a tributary of the Verde River in central Arizona. Preburn precipitation, streamflow, and sediment data were collected for the adjacent paired basins for 8 years from October 1965 to September 1973. Part of the East Fork Sycamore Creek basin was burned on October 31, 1981. Postburn precipitation, streamflow, and sediment data were collected for 3.5 years from October 1982 to May 1986 when the study was discontinued because the runoff and sediment yield appeared to return to preburn levels.

The possibility that some storms in 1985 bypassed the East Fork basin may have a small effect on the results of the study. For the first period of storm of the 1985 water year, the measured amount of precipitation was less for the East Fork basin than for the West Fork basin, and the resulting streamflow for East Fork was less than expected. The effect of the burn probably was masked in early 1985 by the small amount of precipitation for the East Fork basin.

Although storm runoff increased in the postburn period, the percentage of increase was less for periods of higher runoff. Little or no effect of the burn on high streamflows also is indicated by the absence of change in magnitude of peak discharges on the East Fork basin in the postburn period.

Examination of the flow-duration curves of daily discharge showed that the frequency at which a given discharge is equaled or exceeded was greater at most discharges. For the first 2 years of the study, a marked increase occurred in the low flow of the East Fork basin and flow was perennial. Before the burn, the flow of the East Fork basin was ephemeral. A change of flow at the two small springs in the East Fork basin was not detected.

The suspended-sediment yield for the East Fork basin was much greater than the suspended-sediment yield for the West Fork basin for the postburn years of 1983 and 1984. The suspended-sediment yield was 24 and 88 times more for the East Fork basin than for the West Fork basin in those 2 years. For 1985 and part of water year 1986, the suspended-sediment yield for the East Fork basin was only 2.8 and 2.6 times greater than that for the West Fork basin. Because analysis of covariance of suspended-sediment yield of the paired basins indicates that the increase in suspended-sediment yield is related to the increase in streamflow, and not to an increase in sediment concentration, the suspended-sediment yield was considered to return to preburn levels 3 years after the burning of the chaparral vegetation.

The measurements of erosion at the hillside transects and the visual inspection of both basins indicated no increase in hillside erosion as a result of the burn. Evidence did not indicate formation of new rills or sloughing of colluvium in the East Fork basin as a result of the bum. Also, aggradation or degradation was not detected along the main channel of East Fork Sycamore Creek at the channel cross sections. However, the absence of measured or observed changes of hillside erosion and channel aggradation and (or) degradation as a result of the burn does not mean that small changes did not occur.

\section{REFERENCES CITED}

Collings, M.R., and Myrick, R.M., 1966, Effects of juniper and pinyon eradication on streamflow from Corduroy Creek basin, Arizona: U.S. Geological Survey Professional Paper 491-B, 12 p.

Davis, E.A., and Pase, C.P., 1977, Root system of shrub live oak-Implications for water yield in Arizona chaparral: Journal of Soil and Water Conservation, v. 32, no. 4, July-August 1977, p. 174.

DeBano, L.F., 1981, Water repellent soils-A state of the art: U.S. Forest Service General Technical Report PSW-46, p. 21.

Draper, N.R., and Smith, Harry, 1981, Applied regression analysis $(2 \mathrm{~d}$ ed.): New York, John Wiley, $709 \mathrm{p}$.

Guy, H.P., 1970, Fluvial sediment concepts: U.S. Geological Survey Techniques of Water-Resources Investigations, book 3, chap. $\mathrm{C} 1,55 \mathrm{p}$. 
Hibbert, A.R., 1981, Opportunities to increase water yield in the southwest by vegetation management, in Interior West Drainage Basin Management Symposium: Washington State University, Spokane, 1980, Proceedings, p. 223-230.

Hibbert, A.R., Davis, E.A., and Brown, T.C., 1975, Managing chaparral for water and other resources in Arizona, in Drainage Basin Management: American Society of Civil Engineers, Irrigation and Drainage Division, Logan, Utah, Proceedings, p. $445-468$.

Hibbert, A.R., Davis, E.A., and Scholl, D.G., 1974, Chaparral conversion potential in Arizona-Part I, Water yield response and effect on other resources: U.S. Forest Service Research Paper RM-126, p. 36.

Hibbert, A.R., and Ingebo, P.A., 1971, Chaparral treatment effects on streamflow, in 15th Annual Arizona Drainage Basin Symposium, Proceedings: Arizona Water Commission Report 1, p. 25-34.

Hirsch, R.M., Scott, R.G., and Wyant, Timothy, 1982, Investigation of trends in flooding in the Tug Fork Basin of Kentucky, Virginia, and West Virginia: U.S. Geological Survey Water-Supply Paper 2203, $37 \mathrm{p}$.

Hjalmarson, H.W., 1975, Possible effects of vegetation conversion on runoff and sediment yield, Sycamore Creek drainage basins, Maricopa County, Arizona-Calibration-period analysis: U.S. Geological Survey Open-File Report 75-425, 77 p.

Inman, R.L., and Conover, W.J., 1983, A modern approach to statistics: New York, John Wiley, $497 \mathrm{p}$.

Linsley, R.K., Kohler, M.A., and Paulhus, J.L.H., 1982, Hydrology for engineers (3d ed.): New York, McGraw-Hill, 508 p.
Novak, C.E., 1985, WRD data reports preparation guide: U.S. Geological Survey special report, 1985 edition, $199 \mathrm{p}$.

Pase, C.P., and Ingebo, P.A., 1965, Burned chaparral to grass-Early effects on water and sediment yields from two granitic soil drainage basins in Arizona, in 9th Annual Arizona Drainage Basin Symposium, Proceedings: Arizona State Land Department, p. 8-11.

Porterfield, George, 1972, Computation of fluvial-sediment discharge: U.S. Geological Survey Techniques of Water-Resources Investigations, book 3 , chap. $\mathrm{C} 3,66 \mathrm{p}$.

Potter, J.H., 1967, Handbook of the engineering sciences: Princeton, New Jersey, D. Van Nostrand Company, Inc., 1347 p.

Riggs, H.C., 1968, Some statistical tools in hydrology: U.S. Geological Survey Techniques of WaterResources Investigations, book 4, chap. A1, p. 39.

Schumann, H.H., 1967, Water resources of lower Sycamore Creek, Maricopa County, Arizona: U.S. Geological Survey open-file report, 54 p.

Sellers, W.D., and Hill, R.H., eds., 1974, Arizona climate 1931-1972: Tucson, University of Arizona Press, 616 p.

Thomsen, B.W., and Baldys, Stanley, III, 1985, Ground-water conditions in and near the Gila River Indian Reservation, south-central Arizona: U.S. Geological Survey Water-Resources Investigations Report 85-4073, 2 sheets.

Thomsen, B.W., and Schumann, H.H., 1968, Water resources of the Sycamore Creek watershed, Maricopa County, Arizona: U.S. Geological Survey Water-Supply Paper 1861, 53 p. 